ESAIM: COCV 21 (2015) 442-464

DOI: $10.1051 / \mathrm{cocv} / 2014033$
ESAIM: Control, Optimisation and Calculus of Variations

www.esaim-cocv.org

\title{
OPTIMAL FEEDBACK CONTROL FOR UNDAMPED WAVE EQUATIONS BY SOLVING A HJB EQUATION*,**
}

\author{
Axel Kröner ${ }^{1}$, Karl Kunisch ${ }^{2}$ and HasnaA Zidani ${ }^{3}$
}

\begin{abstract}
An optimal finite-time horizon feedback control problem for (semi-linear) wave equations is presented. The feedback law can be derived from the dynamic programming principle and requires to solve the evolutionary Hamilton-Jacobi Bellman (HJB) equation. Classical discretization methods based on finite elements lead to approximated problems governed by ODEs in high dimensional spaces which makes the numerical resolution by the HJB approach infeasible. In the present paper, an approximation based on spectral elements is used to discretize the wave equation. The effect of noise is considered and numerical simulations are presented to show the relevance of the approach.
\end{abstract}

Mathematics Subject Classification. 49J20, 35L05, 49J15.

Received December 17, 2013.

Published online March 9, 2015.

\section{INTRODUCTION}

In this paper we consider optimal feedback control for one-dimensional (semi-linear) wave equations. Let $\Omega \subset \mathbb{R}^{1}$ be an open interval, $T>0$. We consider control problems of the following type

$$
\left\{\begin{aligned}
\min _{u \in \mathcal{U}} J(u) & =\int_{0}^{T} l(\mathbf{y}(s), u(s)) \mathrm{e}^{-\mu s} \mathrm{~d} s \\
\frac{\mathrm{d}}{\mathrm{d} s} \mathbf{y}(s) & =\mathbf{F}(\mathbf{y}(s), u(s)) \\
\mathbf{y}(0) & =y_{0}
\end{aligned}\right.
$$

\footnotetext{
Keywords and phrases. Optimal control, wave equation, Hamilton-Jacobi Bellman equation, spectral elements.

* The work of the second author was supported in part by the Fonds zur Förderung der wissenschaftlichen Forschung under SFB 32, Mathematical Optimization and Applications in the Biomedical Sciences.

** The work of the third author was partially supported by the EU under the 7th Framework Program Marie Curie Initial Training Network FP7-PEOPLE-2010-ITN, SADCO project, GA number 264735-SADCO.

1 Johann Radon Institute for Computational and Applied Mathematics (RICAM), Austrian Academy of Sciences, Altenberger Straße 69, 4040 Linz, Austria. axel.kroener@oeaw.ac.at

2 University of Graz, Institute of Mathematics and Scientific Computing, Heinrichstr. 36, 8010 Graz, Austria and Johann Radon Institute for Computational and Applied Mathematics (RICAM), Austrian Academy of Sciences, Altenberger Straße 69, 4040 Linz, Austria. karl.kunisch@uni-graz.at.

3 INRIA Saclay \& ENSTA ParisTech, 828 Boulevard des Marchaux, 91762 Palaiseau cedex, France. Hasnaa.Zidani@ensta.fr
} 
for given distributed costs $l: L^{2}(\Omega) \times L^{2}(\Omega) \rightarrow \mathbb{R}$, discount factor $\mu$, set of admissible controls $\mathcal{U}$, dynamics $\mathbf{F}$ given by a (semi-linear) wave equation, and initial point $y_{0}$ at time $t=0$. The state $\mathbf{y}$ is defined as the solution of the wave equation under the control $u$. A precise definition is given in the next section.

Although there are many publications for numerical methods for open-loop optimal control problems with partial differential equations, there are only few results on numerical methods for closed-loop optimal control of partial differential equations which are conceived for nonlinear problems. Riccati-based methods and their numerical approximations for the linear regulator problem in the context of infinite dimensional systems have received a significant amount of attention in the past. The linear-quadratic regulator for infinite dimensional, second order (in time) linear oscillators is considered in [19]. In [3] an approximation framework for the computation (in finite dimensional spaces) of Riccati operators is presented that converges to the Riccati operator for the linear regulator problem for a large class of parabolic systems. In [20] a hybrid method for computing feedback gains in linear quadratic regulator problems is analyzed which is in particular appropriate when used with large dimensional systems like control systems for partial differential equations and in [27] a regularity result for solutions of Riccati equation is shown which is essential to obtain a rate of convergence for numerical approximations. A review article on continuous and approximation theory for Riccati equations can be found in [34].

For recent results on Riccati-based methods in the context of control of partial differential equations see, e.g., [36], where an explicit solution for Riccati equations arising in transport theory is considered. In [4] the authors study a Riccati approach for the two dimensional Navier Stokes equation, and in $[13,39]$ the authors consider feedback stabilization for Burgers and Navier-Stokes equations.

For optimal feedback control of partial differential equations based on Hamilton-Jacobi Bellman (HJB) equations there exists only few publications. Here we mention some of them. In [16] internal approximation schemes for optimal control problems in Hilbert spaces are considered and conditions for convergence of the approximate value function are given. In [21] HJB equations for optimal control problems of semi-linear parabolic equations are analyzed. For optimal feedback control of the Burgers equation using proper orthogonal decomposition (POD) we refer to [31,33]. For an optimal feedback control approach of an advection-diffusion equation with an adaptive POD method see [1].

For existing results in the context of feedback control of the wave equation we refer to [19], for the strongly damped wave equation and the Timoshenko beam to [24,25]. For open-loop control of the wave equation see $[18,23,29,30,32]$ and the references cited therein.

In this paper we use a feedback law for the semi-discrete problem of (1.1) which is based on the solution of the corresponding HJB equation. We study the stabilizing effect of the feedback control with respect to noise effecting the dynamics or the observation for linear and nonlinear equations. For validation purposes we compare the results with a linearized Riccati approach. We observe that in the case of the linear wave equation the HJB and the Riccati approach lead to similar results. In case of a nonlinear equation the Riccati approach may fail while the HJB approach still stabilizes the system.

The discretization of partial differential equations in space usually leads to high dimensional dynamical systems and it is therefore very challenging to solve the corresponding HJB equation (so-called curse of dimensionality). In the last decades, several theoretical and numerical developments in HJB theory led to powerful and efficient numerical approaches that can be used for control problems up to 6-dimensional problems $[2,7,8,10,15,37,40]$. For higher dimensional problems, various approaches have been studied in the literature, including model reduction or advanced numerical schemes as, e.g., sparse grids, see [11]. Finally, we also point out that a general introduction for Hamilton-Jacobi Bellman equations and the numerical treatment can be found in the monograph [6].

In our approach we overcome the difficulty (curse of dimensionality) by discretizing the wave equation with spectral elements in space. In many situations already few basis functions are sufficient to obtain a useful approximation. This choice of basis functions leads to diagonal stiffness and mass matrices which allow in certain situations a decomposition of the originally problem in lower dimensional subproblems which can be solved in parallel. 
The paper is organized as follows. In Section 2 we formulate the continuous control problem, in Section 3 we present a semi-discretization in space of (semi-linear) wave equations and formulate the DPP and the corresponding HJB equation, in Section 4 we describe the decomposition in subproblems in case of optimal control of the linear wave equation, in Section 5 we formulate the discrete scheme for solving the HJB equation, in Section 6 we formulate the numerical feedback law, and in Section 7 we present some numerical examples.

\section{ThE CONTROL PROBLEM}

In this section we introduce the control problem in its functional analytic setting. Let

$$
\mathbf{A}: H_{0}^{1}(\Omega) \times L^{2}(\Omega) \rightarrow L^{2}(\Omega) \times H^{-1}(\Omega), \quad \mathbf{A}=\left(\begin{array}{cc}
0 & \text { id } \\
c \Delta & 0
\end{array}\right)
$$

with the negative Laplacian $(-\Delta): H_{0}^{1}(\Omega) \rightarrow H^{-1}(\Omega)$, parameter $c>0$, and identity map id: $L^{2}(\Omega) \rightarrow L^{2}(\Omega)$. We consider semi-linear wave equations (written as a first order system in time) of the following type

$$
\left\{\begin{aligned}
\frac{\mathrm{d}}{\mathrm{d} s} \mathbf{y}(s)-\mathbf{A y}(s)-g\left(\mathbf{y}^{1}(s)\right) & =\bar{f}(s), \quad s \in(0, T), \\
\mathbf{y}(0) & =y_{0}
\end{aligned}\right.
$$

with solution $\mathbf{y}=\left(\mathbf{y}^{1}, \mathbf{y}^{2}\right)^{T}, t \in[0, T), T>0, \bar{f}=(0, f(\cdot))^{T}, f \in L^{2}\left(L^{2}(\Omega)\right)$, initial point $y_{0} \in H_{0}^{1}(\Omega) \times L^{2}(\Omega)$, and nonlinearity

$$
g: L^{2}(\Omega) \rightarrow\{0\} \times L^{2}(\Omega), \quad z \mapsto\left(0, g_{2}(z)\right)^{T}
$$

with

$$
\|g(z)-g(w)\|_{L^{2}(\Omega)} \leq L_{g}\|z-w\|_{L^{2}(\Omega)} \quad \forall z, w \in L^{2}(\Omega)
$$

for Lipschitz constant $L_{g}>0$. We assume that there exists a unique bounded solution in $C\left([0, T] ; H_{0}^{1}(\Omega) \times\right.$ $L^{2}(\Omega)$ ).

Remark 2.1. For the linear wave equation (i.e. $g \equiv 0)$ there exists a unique solution $\mathbf{y} \in C\left([0, T] ; H_{0}^{1}(\Omega) \times\right.$ $L^{2}(\Omega)$ ) (see [35], p. 275). Existence and uniqueness for the semi-linear wave equation can be shown under further conditions on the nonlinear source term $g$, see [41]. The Lipschitz continuity assumption (2.2) of $g$ will be used for the HJB approach in next section.

To set up a control problem we introduce the set of admissible controls

$$
\mathcal{U}=L^{2}((0, T) ; U)
$$

with

$$
U=\left\{u \in \mathbb{R}^{m} \mid u_{a}^{i} \leq u_{i} \leq u_{b}^{i} \text { for } i=1, \ldots, m\right\}
$$

and $u_{a}^{i}, u_{b}^{i} \in \mathbb{R}, i=1, \ldots, m, m \in \mathbb{N}$. Further, we define the operator

$$
\mathbf{B}: \mathbb{R}^{m} \rightarrow\{0\} \times L^{2}(\Omega), \quad \mathbf{B} u=(0, \tilde{u})^{T}, \quad \tilde{u}=\sum_{i=1}^{m} u_{i} \sin (\pi i x),
$$

i.e. for every input $u \in \mathcal{U}$, the function $\mathbf{B}$ is a control of the amplitudes of the basis modes. Now, consider the distributed cost given by:

$$
l: L^{2}(\Omega)^{2} \times \mathbb{R}^{m} \rightarrow \mathbb{R}, \quad l(z, u)=\frac{1}{2}\left\|C z-y_{\mathrm{d}}\right\|_{L^{2}(\Omega)}^{2}+\frac{\alpha}{2}\|u\|^{2}
$$

for parameter $\alpha>0, C=(\mathrm{id}, 0)$, and a given desired state $y_{\mathrm{d}} \in L^{2}(\Omega)$, respectively. Here, $\|\cdot\|$ denotes the Euclidean norm. 
Then the control problem is defined as

$$
\left\{\begin{array}{c}
\min _{u \in \mathcal{U}} \int_{0}^{T} l(\mathbf{y}(s), u(s)) \mathrm{e}^{-\mu s} \mathrm{~d} s, \\
\frac{\mathrm{d}}{\mathrm{d} s} \mathbf{y}(s)=\mathbf{F}(\mathbf{y}(s), u(s)), \\
\mathbf{y}(0)=y_{0}
\end{array}\right.
$$

for $y_{0} \in Y^{1}$, a discount factor $\mu \geq 0$, and operator $\mathbf{F}$ defined by

$$
\mathbf{F}: Y^{1} \times L^{2}(\Omega) \rightarrow L^{2}(\Omega) \times L^{2}(\Omega), \quad \mathbf{F}(x, u)=\mathbf{A} x+\mathbf{B} u+g\left(x^{1}\right)
$$

with $x=\left(x_{1}, x_{2}\right)^{T} \in Y^{1}$.

In the sequel, we assume that for each control $u \in \mathcal{U}$, there exists a unique state $\mathbf{y}$ solution of (2.7).

\section{SEMi-Discrete FORMUlation AND HJB EQUATion}

In this section a semi-discretization in space of the wave equation is presented. This allows to formulate the dynamic programming principle for the corresponding semi-discrete control problem and to derive a HJB equation whose solution is the value function of the semi-discrete problem.

The curse of dimensionality (i.e. the infeasibility to solve high dimensional HJB equations) requires to consider semi-discrete approximations with low dimensional discrete dynamical systems. This is the case when discretizing the wave equation by spectral elements in space. Then the obtained approximated control problem governed by ordinary differential equations has a reasonable number of state variables which allow the problem to be solved by a low dimensional HJB equation.

\subsection{Discretization in space}

For the semi-discretization in space we make the following ansatz. Let the state be given by $y^{h}=\left(y_{1}^{h}, y_{2}^{h}\right)$ with

$$
y_{1}^{h}(x, t)=\sum_{k=1}^{N} y_{1, k}^{h}(t) \varphi_{k}(x), \quad y_{2}^{h}(x, t)=\sum_{k=1}^{N} y_{2, k}^{h}(t) \varphi_{k}(x)
$$

for given basis functions $\varphi_{k} \in H_{0}^{1}(\Omega), k=1, \ldots, N$, and set

$$
y^{h}:=\left(y_{1,1}, \ldots, y_{1, N}, y_{2,1}, \ldots, y_{2, N}\right)^{T} \in L^{2}\left((0, T) ; \mathbb{R}^{2 N}\right) .
$$

In the following we will always assume that $N=m$.

As mentioned above it is important to choose a low order of approximation $N$, since the HJB equation that will be associated to the approximated control problem will be in a $2 N$-dimensional space, see Section 3.3 where we will specify the choice of the basis functions in more detail.

Further, we define the stiffness and mass matrices

$$
\begin{aligned}
A_{h} & =\left(\left(\nabla \varphi_{i}, \nabla \varphi_{j}\right)_{L^{2}(\Omega)}\right)_{i=1, \ldots, N, j=1, \ldots, N}, \\
M_{h} & =\left(\left(\varphi_{i}, \varphi_{j}\right)_{L^{2}(\Omega)}\right)_{i=1, \ldots, N, j=1, \ldots, N}
\end{aligned}
$$

with respect to the basis $\varphi_{i}, i=1, \ldots, N$.

We introduce the discrete dynamics

$$
\mathbf{F}_{h}: \mathbb{R}^{2 N} \times \mathbb{R}^{N} \rightarrow \mathbb{R}^{2 N}, \quad \mathbf{F}_{h}(z, u)=\mathbf{M}_{h}^{-1}\left(\mathbf{A}_{h} z+\mathbf{B}_{h} u+g_{h}(z)\right)
$$


with matrices

and nonlinearity

$$
\mathbf{M}_{h}=\left(\begin{array}{cc}
M_{h} & 0 \\
0 & M_{h}
\end{array}\right), \quad \mathbf{A}_{h}=\left(\begin{array}{cc}
0 & M_{h} \\
-c A_{h} & 0
\end{array}\right), \quad \mathbf{B}_{h}=\left(\begin{array}{c}
0 \\
M_{h}
\end{array}\right)
$$

$$
g_{h}(z)=\left(0, g_{h, 2}(z)\right)^{T}, \quad g_{h, 2}(z)=\left(\left(\left(g_{2}\left(\sum_{k=1}^{N} z_{k} \varphi_{k}\right), \varphi_{i}\right)_{L^{2}(\Omega)}\right)_{i=1, \ldots, N}\right)
$$

with $z=\left(z_{1}, \ldots, z_{N}, z_{N+1}, \ldots, z_{2 N}\right)^{T}$ and the discrete distributed cost

$$
l_{h}: \mathbb{R}^{2 N} \times \mathbb{R}^{N} \rightarrow \mathbb{R}, \quad l_{h}(z, u)=\frac{1}{2}\left(C_{h} z-y_{\mathrm{d}}^{h}\right)^{T} M_{h}\left(C_{h} z-y_{\mathrm{d}}^{h}\right)+\frac{\alpha}{2} u^{T} u
$$

for $\alpha>0, y_{\mathrm{d}}^{h} \in \mathbb{R}^{N}$, where $y_{\mathrm{d}}^{h}$ is the vector containing the coordinates of $y_{\mathrm{d}}$ with respect to the spectral basis functions, $C_{h} \in \mathbb{R}^{2 N \times 2 N}$ with $C_{h}=\left(\operatorname{id}_{N}, 0\right)^{T}$. Here, $\operatorname{id}_{N}$ denotes the identity on $\mathbb{R}^{N}$. The Lipschitz continuity of $g$ implies the Lipschitz continuity of $g_{h}$. The semi-discrete control problem is given by

$$
\left\{\begin{aligned}
\inf _{u \in \mathcal{U}} J^{h}(u) & =\int_{0}^{T} l_{h}\left(y_{x, t}^{h}(s), u(s)\right) \mathrm{e}^{-\mu s} \mathrm{~d} s, \quad \text { s.t. } \\
\frac{\mathrm{d}}{\mathrm{d} s} y^{h}(s) & =\mathbf{F}_{h}\left(y^{h}(s), u(s)\right) \quad \text { for } s \in(0, T), \\
y^{h}(0) & =y_{0}^{h},
\end{aligned}\right.
$$

for initial value $y_{0}^{h} \in \mathbb{R}^{2 N}$. The initial state $y_{0}^{h}$ is the projection of $y_{0}$ on the finite dimensional space $V_{h} \times V_{h}$ with $V_{h}=\operatorname{span}\left(\varphi_{1}, \ldots, \varphi_{N}\right)$.

To simplify the notation we will henceforth omit the index $h$.

\subsection{Dynamic programming principle and Hamilton-Jacobi Bellman equation}

For $x \in \mathbb{R}^{2 N}$ and $t \in[0, T)$, we consider the parameterized control problem:

$$
\left\{\begin{array}{l}
\inf _{u \in \mathcal{U}} \int_{t}^{T} l_{h}\left(y_{x, t}(s), u(s)\right) \mathrm{e}^{-\mu s} \mathrm{~d} s, \\
\frac{\mathrm{d}}{\mathrm{d} s} y_{x, t}(s)=\mathbf{F}_{h}\left(y_{x, t}(s), u(s)\right) \quad \text { for } s \in(t, T), \\
y_{x, t}(t)=x .
\end{array}\right.
$$

In the above problem, the notation $y_{x, t}$ denotes the solution of the ODE system that starts in the position $x$ at time $t$. To the family of problems $\left(\mathcal{P}_{x, t}\right)$, we consider the value function $v: \mathbb{R}^{2 N} \times[0, T] \rightarrow \mathbb{R}$ that associates to each initial condition $(x, t)$ the infimum value of the problem $\left(\mathcal{P}_{x, t}\right)$ :

$$
v(x, t)=\inf \left(\mathcal{P}_{x, t}\right) .
$$

The value function $v$ satisfies the dynamic programming principle (DPP), see [6], pp. 154-155

$$
v(x, t)=\inf _{u \in \mathcal{U}}\left(\int_{t}^{\tau} \mathrm{e}^{\mu(t-s)} l_{h}\left(y_{x, t}(s), u(s)\right) \mathrm{d} s+\mathrm{e}^{\mu(t-\tau)} v\left(y_{x, t}(\tau), \tau\right)\right),
$$

for $\tau \in[t, T], t \in[0, T]$.

From the DPP we derive the value function $v$ is given as the viscosity solution of the evolutionary Hamilton-Jacobi Bellman (HJB) equation (cf. [6], p. 155),

$$
\left\{\begin{aligned}
-\partial_{t} v(x, t)+\mu v(x, t)+\mathcal{H}\left(x, \nabla_{x} v(x, t)\right)=0 & & (x, t) \in \mathbb{R}^{2 N} \times(0, T), \\
v(x, T)=0 & & x \in \mathbb{R}^{2 N},
\end{aligned}\right.
$$


with Hamiltonian

$$
\begin{aligned}
& \mathcal{H}: \mathbb{R}^{2 N} \times \mathbb{R}^{2 N} \longrightarrow \mathbb{R}, \\
& \mathcal{H}(x, p)=\sup _{u \in U}\left(-\mathbf{F}_{h}(x, u)^{T} p-l_{h}(x, u)\right) .
\end{aligned}
$$

Moreover, from [26] we know that $v$ is the unique viscosity solution of (3.7) with quadratic growth.

Finally, let us point out that by straightforward calculations, the Hamiltonian can be reformulated as:

$$
\begin{aligned}
\mathcal{H}(x, p)= & \left(-x^{T}\left(\mathbf{M}_{h}^{-1} \mathbf{A}_{h}\right)^{T} p-\left(\mathbf{M}_{h}^{-1} g\left(x^{1}\right)\right)^{T} p-u(p)^{T}\left(\mathbf{M}_{h}^{-1} \mathbf{B}_{h}\right)^{T} p\right. \\
& \left.-\left(\frac{1}{2}\left(C_{h} x-y_{\mathrm{d}}^{h}\right)^{T} M_{h}\left(C_{h} x-y_{\mathrm{d}}^{h}\right)\right)-\frac{\alpha}{2} u(p)^{T} u(p)\right)
\end{aligned}
$$

with

$$
u(p):=\operatorname{argmax}_{u \in U}\left(-\mathbf{F}_{h}(x, u)^{T} p-l_{h}(x, u)\right)=P_{\left[u_{a}, u_{b}\right]}\left(-\frac{1}{\alpha} \mathbf{B}_{h}^{T} \mathbf{M}_{h}^{-T} p\right)
$$

for any $p \in \mathbb{R}^{2 N}$, and projection $P_{\left[u_{a}, u_{b}\right]}$ defined by

$$
P_{\left[u_{a}, u_{b}\right]}(z)=\hat{z}, \quad \hat{z}_{i}=\min \left(u_{b}^{i}, \max \left(u_{a}^{i}, z_{i}\right)\right)
$$

for $z \in \mathbb{R}^{2 N}$. In case of inactive control constraints this reduces to

$$
\mathcal{H}(x, p)=-x^{T}\left(\mathbf{M}_{h}^{-1} \mathbf{A}_{h}\right)^{T} p-\left(\mathbf{M}_{h}^{-1} g\left(x^{1}\right)\right)^{T} p-\left(\frac{1}{2}\left(C_{h} x-y_{\mathrm{d}}^{h}\right)^{T} M_{h}\left(C_{h} x-y_{\mathrm{d}}^{h}\right)\right)+\frac{1}{2 \alpha} p^{T} \mathcal{B B}^{T} p
$$

with $\mathcal{B}=\mathbf{M}_{h}^{-1} \mathbf{B}_{h}$.

\subsection{Spectral elements}

In general it is very challenging to solve the HJB (3.7) in high dimensions. To obtain a low dimensional problem we use spectral elements for the discretization of the wave equation in space. This allows to use only few basis functions to obtain an appropriate approximation. Spectral elements play an important role and are widely used for the numerical discretization of partial differential equations, see, e.g. $[14,22,38]$ and in the context of optimal control [17]. To discretize the wave equation by spectral elements we specify the domain $\Omega=(0,1)$ and choose the basis functions by

$$
\varphi_{k}(x)=\sin (k \pi x), \quad k=1, \ldots, N, \quad x \in \Omega .
$$

The corresponding mass and stiffness matrix are diagonal and are given by

$$
M_{h}=\operatorname{diag}\left((1 / 2)_{k=1, \ldots, N}\right), \quad A_{h}=\operatorname{diag}\left(\left(\frac{1}{2}(k \pi)^{2}\right)_{k=1, \ldots, N}\right) .
$$

The following numerical example shows, that for the corresponding open-loop control problem only few spectral basis functions are necessary to obtain a good approximation.

Example 3.1. We consider problem (2.7) and allow that the distributed costs also depend on time. We choose $T=1, \Omega=(0,1), \mu=0, c=1$, and the distributed costs as

$$
\begin{aligned}
& l: L^{2}(\Omega) \times L^{2}(\Omega) \times[0, T] \rightarrow \mathbb{R}, \\
& l(z, u, t)=\frac{1}{2}\left\|z-y_{\mathrm{d}}(t)\right\|_{L^{2}(\Omega)}^{2}+\frac{\alpha}{2}\|u\|_{L^{2}(\Omega)}^{2}
\end{aligned}
$$


with $\alpha=1$, and the desired state by

$$
\begin{aligned}
y_{\mathrm{d}}(z, t)= & (t-T)^{4} z^{3}(1-z)^{3}+24 z^{3}(1-z)^{3}-12(t-T)^{2}\left(6 z(1-z)^{3}-18 z^{2}(1-z)^{2}\right. \\
& \left.+6 z^{3}(1-z)\right)-12(t-T)^{2}\left(6 z(1-z)^{3}-18 z^{2}(1-z)^{2}+6 z^{3}(1-z)\right) \\
& +(t-T)^{4}\left(-18(1-z)^{2}+216 z(1-z)-54(1-z)^{2}-72 z^{2}\right) .
\end{aligned}
$$

The initial values are given by

$$
x(z)=\left(T^{4} z^{3}(1-z)^{3},-4 T^{3} z^{3}(1-z)^{3}\right)^{T} .
$$

The constraints on the control are chosen in such a way that they remain inactive. Then the exact state is given by

$$
y^{*}(z, t)=\left((t-T)^{4} z^{3}(1-z)^{3}, 4(t-T)^{3} z^{3}(1-z)^{3}\right)^{T} .
$$

For $N=5$ spectral basis functions the error between the exact solution $y^{*}$ and the numerical approximation $\tilde{y}$ is $\left\|\tilde{y}-y^{*}\right\|_{L^{2}\left(L^{2}(\Omega)\right)} \approx 1.29 \times 10^{-4}$. For time discretization a Crank Nicolson scheme is applied with temporal mesh parameter $k=1.0 \times 10^{-4}$. This example shows that already few spatial basis functions lead to a good approximation of the state.

Remark 3.2. As already known and mentioned earlier in the paper, the theory of the HJB approach is valid in any dimension. However, in practice, the implementation of the method in high dimension requires, in general, a huge numerical effort (data storage and computing time).

Several works have dealt with model reduction of optimal control problems governed by partial differential equations leading to a reduced controlled system that can be studied numerically by using the HJB approach, see, e.g., [31,33], where proper orthogonal decomposition (POD) is applied to reduce the dimension of the underlying dynamical system, and in [1], where for adaptively chosen small time horizons the dynamical system is reduced by POD.

Note also that it would be interesting to reduce the dimension not in the definition of the control problem, but in the HJB equation associated to it. However, for such a nonlinear PDE the analysis of reduction techniques is still an open problem, see [11] for a method based on sparse grids.

\section{Decomposition in two Dimensional SUbProblems}

The discretization by spectral elements leads to diagonal mass and stiffness matrices, i.e. the set of basis functions is an orthogonal basis with respect to the $L^{2}(\Omega)$ - and $H_{0}^{1}(\Omega)$-inner product. In certain situations this allows to decompose the $2 N$-dimensional HJB equation in $N$ 2-dimensional decoupled HJB equations which can be solved in parallel. In this section we assume that the problem is of linear-quadratic type with possibly constraints on the control, i.e.

$$
\left\{\begin{aligned}
\min _{u \in \mathcal{U}} J^{h}(x, t, u) & =\int_{t}^{T} l_{h}\left(y_{x, t}(s), u(s)\right) \mathrm{e}^{-\mu s} \mathrm{~d} s, \quad \text { s.t. } \\
\frac{\mathrm{d}}{\mathrm{d} s} y_{x, t} & =\mathbf{M}_{h}^{-1}\left(\mathbf{A}_{h} y_{x, t}+\mathbf{B}_{h} u\right) \\
y_{x, t}(t) & =x
\end{aligned}\right.
$$

We introduce $N$ subproblems defined, for $i=1, \ldots, N$ by

$$
\left\{\begin{aligned}
v_{i}(\hat{z}, t) & =\min _{u(\cdot) \in \mathcal{U}_{i}} \frac{1}{2} \int_{t}^{T}\left(\frac{1}{2}\left\|z_{i}^{1}(s)\right\|^{2}+\alpha u^{2}(s)\right) \mathrm{e}^{-\mu s} \mathrm{~d} s, \\
\dot{z}_{i}(s) & =A_{i} z_{i}(s)+B u(s), \\
z_{i}(t) & =\hat{z},
\end{aligned}\right.
$$


for $z_{i}=\left(z_{i}^{1}, z_{i}^{2}\right)^{T}$ and with $\mathcal{U}_{i}=L^{2}\left((0, T) ; U_{i}\right)$ for $U_{i}=\left\{u \in \mathbb{R} \mid u_{a}^{i} \leq u \leq u_{b}^{i}\right\}$ and

$$
A_{i}=\left(\begin{array}{rr}
0 & 1 \\
a_{i} & 0
\end{array}\right), \quad B=\left(\begin{array}{l}
0 \\
1
\end{array}\right)
$$

and where $a_{i}=\left(\mathbf{M}_{h}^{-1} \mathbf{A}_{h}\right)_{i i}$. For every $i=1, \ldots, N$, the value functions $v_{i}$ of the subproblem (4.2) satisfy the following HJB equation:

$$
\left\{\begin{aligned}
-\partial_{t} v_{i}(z, t)+\mu v_{i}(z, t)+\mathcal{H}_{i}\left(z, \nabla_{z} v_{i}(z, t)\right)=0 & & (z, t) \in \mathbb{R}^{2} \times(0, T), \\
v_{i}(z, T)=0 & & z \in \mathbb{R}^{2}
\end{aligned}\right.
$$

with $\mathcal{H}_{i}(z, p):=\sup _{u \in U_{i}}\left(-\left(A_{i} z+B u\right)^{T} p-\frac{1}{2}\left(\frac{1}{2}\|z\|^{2}+\alpha u^{2}\right)\right)$ for $z, p \in \mathbb{R}^{2}$. There holds the following relation between the value function of problem (4.1) and the value functions of the subproblems (4.2).

Theorem 4.1. Let $v_{i}$ be the solution of the subproblem (4.2) for $i=1, \ldots, N$ and let $v$ be the solution of (4.1). Then there holds the relation

$$
v(x, t)=\sum_{i=1}^{N} v_{i}\left(\left[\begin{array}{c}
x_{i} \\
x_{i+N}
\end{array}\right], t\right) \quad \text { for every } t \in(0, T), x=\left[\begin{array}{c}
x_{1} \\
\vdots \\
x_{2 N}
\end{array}\right] \in \mathbb{R}^{2 N} .
$$

Proof. The arguments are straightforward since in the present case (when $g \equiv 0$ ) the controlled system can be split into $N$ decoupled systems. Also the minimisation over the control set $\mathcal{U}$ can be split into $\mathrm{N}$ independent minimisation problems.

The above result means that instead of solving (3.7), one can solve $N$ independent 2-dimensional HJB equations (4.3) with different data. These computations can be done in parallel, which has as a consequence a significant reduction of the computational effort (in terms of data storage and of cpu time).

Remark 4.2. It is well-known that in general the stiffness matrix for spectral elements is ill-conditioned. By the decomposition the drawback of this property is reduced since in every subproblem only two dimensional matrices are considered.

\section{Discretization of the HJB}

Several discretization schemes for HJB equations have been studied in the literature. For an overview on finite differences schemes, we refer to [37]. Another recent reference [15] gives an overview on semi-Lagrangian schemes and the features of this class of methods. For a finite element approach we refer to [28], and for discontinuous Galerkin methods to [7]. A class of anti-diffusive methods has been studied in [8,12]. Here, we use a finite difference scheme method, more precisely, an ENO scheme for space discretization is coupled with a Runge-Kutta time discretization scheme of second order.

\subsection{Numerical scheme}

Following $[10,37]$ we shortly recall the scheme for a given continuous Hamiltonian $\mathcal{H}: \mathbb{R}^{2 N} \times \mathbb{R}^{2 N} \rightarrow \mathbb{R}$. For given temporal mesh parameter $\Delta t$ and spatial mesh parameter $\Delta x=\left(\Delta x_{1}, \ldots, \Delta x_{2 N}\right)$ we define a spatial mesh

$$
\mathcal{G}=\left\{x_{I}=\left(I_{1} \Delta x_{1}, \ldots, I_{2 N} \Delta x_{2 N}\right) \text { for } I \in \mathbb{Z}^{2 N}\right\}
$$

and temporal mesh

$$
t=t_{0}<t_{1}<\ldots<t_{M}=T, \quad t_{j+1}-t_{j}=\Delta t, \quad j=0, \ldots, M_{T}-1, \quad M_{T} \in \mathbb{N} .
$$


Further, we introduce difference quotients for $x \in \mathcal{G}$ by

$$
D^{+} v(x)=\left(D_{x_{1}}^{+} v(x), \ldots, D_{x_{2 N}}^{+} v(x)\right), \quad D^{-} v(x)=\left(D_{x_{1}}^{-} v(x), \ldots, D_{x_{2 N}}^{-} v(x)\right)
$$

with

$$
D_{x_{i}}^{ \pm} v\left(x_{I}\right)= \pm \frac{v\left(x_{I^{i, \pm}}\right)-v\left(x_{I}\right)}{\Delta x}
$$

and $I^{k, \pm}=\left(i_{1}, \ldots, i_{k-1}, i_{k} \pm 1, i_{k+1}, \ldots, i_{2 N}\right)$. Then the Lax-Friedrichs scheme for the HJB equation reads as follows: $v_{I}^{M}=0$ for every $I \in \mathbb{Z}^{2 N}$, and

$$
v_{I}^{n-1}=v_{I}^{n}-\Delta t \mu v_{I}^{n}-\Delta t \mathcal{H}_{L F}\left(x_{I}, D^{+} v^{n}\left(x_{I}\right), D^{-} v^{n}\left(x_{I}\right)\right) \quad \text { for } n=M, \ldots, 1
$$

with Lax-Friedrichs Hamiltonian

$$
\mathcal{H}_{L F}\left(x, p^{+}, p^{-}\right)=\mathcal{H}\left(x, \frac{p^{+}+p^{-}}{2}\right)-\sum_{i=1}^{2 N} \frac{C_{i}(x)}{2}\left(p_{i}^{+}-p_{i}^{-}\right)
$$

and the stabilizing functions $C_{i}(x)$ satisfying

$$
\max _{p \in \mathbb{R}^{2 N}}\left|\frac{\partial \mathcal{H}}{\partial p_{i}}(x, p)\right| \leq C_{i}(x)
$$

for $i=1, \ldots, 2 N$. It is well-known that the Lax-Friedrichs scheme is monotone and the convergence is of first order as far as the CFL condition

$$
k \max _{x \in \mathcal{G}}\left(\sum_{i=1}^{2 N} \frac{C_{i}(x)}{\Delta x_{i}}\right) \leq 1
$$

is satisfied.

An ENO scheme can be obtained by considering a variant of the LF scheme

$$
v_{I}^{n-1}=v_{I}^{n}-\Delta t \mu v_{I}^{n}-\Delta t \mathcal{H}_{L F}\left(x_{I}, \widetilde{D}^{+} v^{n}\left(x_{I}\right), \widetilde{D}^{-} v^{n}\left(x_{I}\right)\right),
$$

where $\widetilde{D}^{ \pm} v^{n}\left(x_{I}\right)$ are higher approximations of the gradient $\partial_{x_{i}} v$ coupled with a Runge-Kutta time discretization scheme of second order, see $[10,37]$. Convergence of this method is not proved but various numerical examples confirm its relevance.

\subsection{Boundary condition for HJB equation on the computational domain}

Since the HJB is defined on the full space we have to define a bounded computational domain $\mathcal{K} \times(0, T)$, with $\mathcal{K} \subset \mathbb{R}^{2 N}$, to solve the problem numerically. For simplicity $\mathcal{K}$ will be chosen as

$$
\mathcal{K}:=\Pi_{i=1}^{2 N}\left[c_{i}, d_{i}\right]
$$

for some $c, d \in \mathbb{R}^{2 N}$ and the considered grid is as follows:

$$
\mathcal{G}_{0}=\left\{x_{I}=\left(c_{1}+\left(I_{1}-1\right) \Delta x_{1}, \ldots, c_{2 N}+\left(I_{2 N}-1\right) \Delta x_{2 N}\right) \text { for } I \in \Pi_{i=1}^{2 N}\left[1, M_{i}\right]\right\},
$$

with $\Delta x_{i}:=\frac{d_{i}-c_{i}}{M_{i}-1}$. However, due the boundedness of $\mathcal{K}$ an appropriate choice of a boundary condition is required to get a well define numerical scheme.

When a strong invariance condition

$$
\mathbf{F}_{h}(x, u) \cdot n(x)<0 \quad \forall x \in \partial \mathcal{K}, \quad \forall u \in U,
$$


(with exterior normal $n$ to the boundary of $\mathcal{K}$ at $x$ ) is satisfied then all trajectories starting in $\bar{D}$ will remain within $\bar{\Omega}$ forever, see [6], p. 485. In this case, there is no need to impose a boundary condition. However, the strong invariance condition is very restrictive and cannot be insured even in the simple case when $g \equiv 0$.

Here, we introduce an artificial boundary condition by setting the second derivative on the boundary to zero:

$$
\partial_{x x} v(x, t)=0 \quad \forall(x, t) \in \partial \Omega \times(0, T) .
$$

This means that at a time step $t_{n}$, for $n=M_{T}, \ldots, 1$, if $x_{I} \in \partial \Omega$, then there exists $i$ such that $I_{i}=1$ or $I_{i}=M_{i}$. In this case the upwind derivatives on the boundary should be defined as:

$$
\begin{cases}D_{x_{i}}^{ \pm} v\left(x_{I}\right)=\frac{v\left(x_{I^{i},+}\right)-v\left(x_{I}\right)}{h_{i}} & \text { if } I_{i}=0 \\ D_{x_{i}}^{ \pm} v\left(x_{I}\right)=-\frac{v\left(x_{I^{i},-}\right)-v\left(x_{I}\right)}{h_{i}} & \text { if } I_{i}=M_{i} .\end{cases}
$$

Since the value function is continuous, this approximation is reasonable. For a more sophisticated treatment of the boundary condition using state constraints we refer to [2].

\section{Reconstruction of the trajectory}

For the reconstruction of the trajectories we formulate a feedback law based on the HJB approach and present different numerical realizations. This approach is applicable on a large class of (nonlinear) problems without modifications of the general setting.

Notice that besides the HJB approach, to study optimal feedback control problems for nonlinear dynamical systems, it is possible in some situations to use a Riccati based approach. In this case, a Riccati feedback operator can be derived from the linearized state equation. This approach has been considered, for instance, in [39] to study boundary control problems governed by Navier Stokes equation. In [13] the authors consider an infinite horizon problem and propose a method using formally a quadratic Taylor approximation of the solution of the HJB equation. For a non-autonomous system that appears in the internal stabilization of Navier Stokes equation to a non-stationary solution a feedback law satisfying a differential Riccati equation can be found in $[5]$.

According to the HJB approach and its associated dynamic programming principle, the optimal feedback control $u_{x}^{*}$ for state $x$ at time $t \in[0, T)$ satisfies

$$
\left.u_{x}^{*}=\underset{u \in \mathcal{U}^{[t, t+\tau]}}{\operatorname{argmin}}\left(\mathrm{e}^{-\mu \tau} v\left(y_{x, t}(t+\tau), t+\tau\right)\right)+\int_{t}^{t+\tau} \mathrm{e}^{\mu(t-s)} l_{h}\left(y_{x, t}(s), u(s)\right) \mathrm{d} s\right)
$$

for $\tau \geq 0$ with $t+\tau \leq T$ (see [6], pp. 149/155), and

$$
\mathcal{U}^{[t, t+\tau]}=\left\{u:[t, t+\tau] \rightarrow \mathbb{R}^{N}|\exists w \in \mathcal{U}: w|_{[t, t+\tau]}=u\right\} .
$$

The feedback operator $\mathbf{K}$ is defined as

$$
\mathbf{K}: \mathbb{R}^{2 N} \times(t, T) \longrightarrow U,(x, \tau) \mapsto \mathbf{K}(x, \tau)=u_{x}^{*}(\tau) .
$$

Once the feedback operator is defined, to reconstruct the optimal trajectory starting in $x_{0}$ at the initial time 0 , one needs just to solve the dynamical system

$$
y^{\prime}(s)=\mathbf{F}_{h}(y(s), \mathbf{K}(y(s), s)), \quad y(0)=x_{0}, \quad s \in(0, T] .
$$

To realize the feedback law (6.2) numerically there are several methods. We give an overview about three of them. Let $v^{\Delta}$ be the discrete value function computed on $\mathcal{G}_{0}$ by the ENO scheme described in Section 5 . 
1. The dynamics and distributed cost are given in a separable form, i.e.

$$
\mathbf{F}(z, u)=F(z)+B u, \quad l(z, u)=j(z)+\frac{\alpha}{2} u^{T} u
$$

with $F: \mathbb{R}^{2 N} \rightarrow \mathbb{R}^{2 N}$, matrix $B \in \mathbb{R}^{2 N \times N}$, and $j: U \rightarrow \mathbb{R}$ as it is the case in the problem under consideration. If the value function satisfy $v \in C^{1}\left(\mathbb{R}^{2 N}\right)$ the feedback operator is determined by the projection of the gradient of the value function on the set of admissible controls (cf. (3.11)), i.e.

$$
\mathbf{K}(x, t)=P_{\left[u_{a}, u_{b}\right]}\left(-\frac{1}{\alpha} B^{T} \nabla_{x} v(x, t)\right) .
$$

Since only an approximation $v^{\Delta}$ of $v$ can be computed, an approximation of the feedback can be given by:

$$
\mathbf{K}^{\Delta}(x, t)=P_{\left[u_{a}, u_{b}\right]}\left(-\frac{1}{\alpha} B^{T} \nabla_{x} v^{\Delta}(x, t)\right),
$$

where the gradient $\nabla_{x} v^{\Delta}$ can be approximated component-by-component by a central difference quotient.

2. The control minimizing the Hamiltonian $\mathcal{H}$ can be saved at every time point and in every mesh point of $\mathcal{G}_{0}$ when solving the HJB equation. For all $x_{I} \in \mathcal{G}_{0}, k \in\left\{0, \ldots, M_{T}\right\}$, we set

$$
\mathbf{K}^{\Delta}\left(x_{I}, t_{k}\right)=\underset{u \in U}{\operatorname{argmax}}\left(-\mathbf{F}_{h}\left(x_{I}, u\right)^{T} \nabla_{x} v^{\Delta}\left(x_{I}, t_{k}\right)+l_{h}\left(x_{I}, u\right)\right),
$$

where $v^{\Delta}$ denotes the discrete value function. This leads to a set of discrete controls $\left\{u_{I}\right\}_{I \in \mathbb{Z}^{2 N}, x_{I} \in \mathcal{K}}$ whose elements correspond to the mesh points. The advantage of this approach is that it gives directly the set of discrete controls without any additional effort. A drawback of this approach is that this set has to be saved and could be very large (as big as the set of all stored values of the value function). Furthermore, to compute a feedback control which corresponds to a state which is not a mesh point in $\mathcal{G}_{0}$ one has to compute the control by an interpolation method. This procedure requires the feedback control operator $\mathbf{K}$ to be continuous, which usually is not the case.

3. In this approach the dynamic programming principle is directly used to reconstruct the trajectories, i.e. using a discrete version of (6.1). We choose the feedback control by

$$
\begin{aligned}
u_{x}^{*}\left(t_{k}\right) & =\underset{u \in U}{\operatorname{argmin}}\left(\left[v^{\Delta}\right]\left(x+\Delta t \mathbf{F}_{h}\left(y_{x, t_{k}}\left(t_{k}\right), u\right)\right)+\Delta t l_{h}\left(y_{x_{I}, t_{k}}\left(t_{k}\right), u\right)\right), \\
& =: \underset{u \in U}{\operatorname{argmin}} G\left(x, y_{x, t_{k}}\left(t_{k}\right), u\right) .
\end{aligned}
$$

Here, $[\cdot]$ is an interpolation of the value function $v^{\Delta}$ on $\mathcal{G}_{0}$. A broadly used method to determine the argmin consists in choosing a finite set $U^{\text {finite }} \subset U$ and computing the argmin with respect to this set by evaluating the expression $G\left(x_{I}, y_{x, t_{k}}\left(t_{k}\right), u\right)$ for all $u \in U^{\text {finite }}$ and taking the control value which minimizes the expression, see [6], p. 475. If the control space is low dimensional, the approach works well. But in case of higher dimensions or a large control set $U^{\text {finite }}$ the approach is only feasible in combination with a strategy which reduces the number of evaluations of $G\left(x_{I}, y_{x, t_{k}}\left(t_{k}\right), u\right)$ in each time step. If the problem can be decomposed as described in Section 4 the number of evaluations reduces strongly.

In all the simulations performed in this paper, we apply the first of these three methods assuming that the value function is sufficiently smooth. This method gives us a direct formula for the feedback control and saves much computational time in comparison to the third option.

\section{NumericAl EXAMPLES}

Several numerical examples are presented studying the stabilizing effect of the feedback control. For solving the HJB equation we use the software package ROC-HJ-Solver, see [9], which is parallized with OpenMP. For the reconstruction of the feedback controls and the corresponding trajectories MATLAB is used. 
In the first three examples we consider an optimal feedback control problem for the linear wave equation and in the fourth one an optimal control problem for a semi-linear wave equation. In all examples we set $\Omega=(0,1)$, $\mu=0, y_{\mathrm{d}}=0$.

\subsection{Linear wave equation - without noise}

In this example we consider the control problem under consideration for the linear wave equation with data

$$
c=0.025, \quad T=8, \quad y_{0}(z)=\left(\sum_{i=1}^{5} 0.3 \sin (i \pi z), 0\right)^{T}, \quad z \in \Omega
$$

and $\alpha=0.1$. To validate the numerical algorithm we compare the trajectory we obtain via the HJB approach with the trajectory via the Riccati approach. The differential Riccati equation for the problem is given by

$$
-P^{\prime}(t)=A^{T} P(t)+P(t) A-P(t) B R^{-1} B^{T} P(t)+Q, \quad P(T)=0
$$

with $Q=M_{h}, R=\alpha \operatorname{id}_{N}, A=\mathbf{M}_{h}^{-1} \mathbf{A}_{h}$, and $B=\mathbf{M}_{h}^{-1} \mathbf{B}_{h}$. The feedback operator for the Riccati approach which maps the optimal state at time $t$ namely $y^{*}(t)$ to the corresponding optimal control $u^{*}(t)$ is given by

$$
K(t)=-R^{-1} B^{T} P(t)
$$

For the reconstruction of the trajectories via the HJB approach we choose the first method presented in Section 6 . The computational domain for the HJB equation is chosen to be $\mathcal{K}=[-1,1]^{10}$. For the temporal discretization we choose the mesh size $\Delta t=2 \times 10^{-3}$. For the spatial discretization we choose a mesh uniform in every spatial direction with the following numbers of discretization points

$$
10 \times 16 \times 30 \times 50 \times 60 \times 10 \times 16 \times 30 \times 50 \times 60
$$

That means for the spatial directions which correspond to higher modes we use more grid points. Further, we decompose the equation in subproblems as described in Section 4. For the discretization of the dynamical system as well as the Riccati equation (7.2) we apply a explicit Euler scheme and set the temporal mesh parameter to $k_{\mathrm{ds}}=10 \Delta t=0.02$.

In Figure 1 we see the first and fifth component of the state $y_{1}$ and velocity $y_{2}$ obtained by the HJB (blue curves) and the Riccati (red curves) approach and the corresponding components of the feedback control of these two approaches. The other components show a corresponding behaviour, therefore we skip the plots here. For the chosen discretization both approaches lead to nearly the same results. If we choose finer spatial mesh sizes when solving the HJB equation the trajectory of the HJB approach approximates the trajectory of the Riccati approach more closely. Further numerical experiments show that for a fixed and sufficiently small temporal mesh size the discretization of the subproblems for the higher modes require a finer spatial mesh to obtain a good approximation. The corresponding state and velocity of the wave equation which correspond to the computed trajectories are shown in Figure 2. The example confirms that the chosen boundary condition described in Section 5.2 leads to appropriate numerical results.

\subsection{Linear wave equation - with noise}

We study the stabilizing effect of the feedback control on the trajectories in presence of random noise which either effects the dynamics or the observation. In this example we choose the parameter $\alpha=0.01$ which will lead to a stronger decrease of the trajectories to zero than in the previous example. 

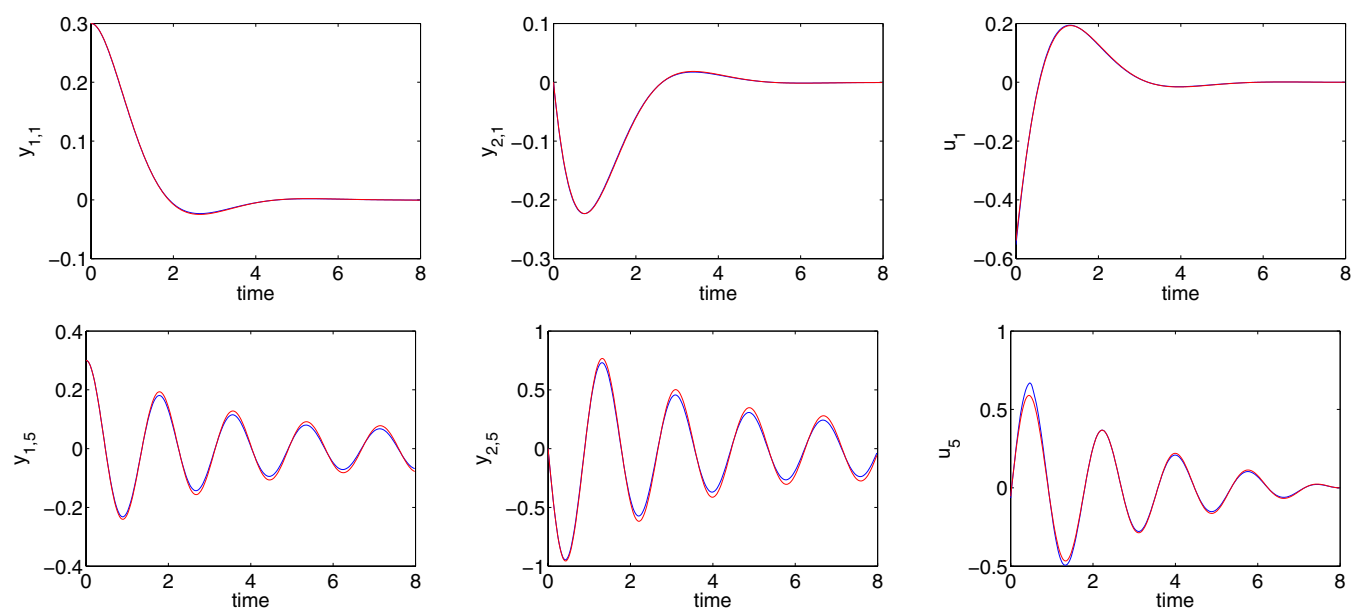

FiguRE 1. Components of the trajectory and the feedback control via HJB (blue) and Riccati (red) approach, $(\alpha=0.1)$. (In color online)
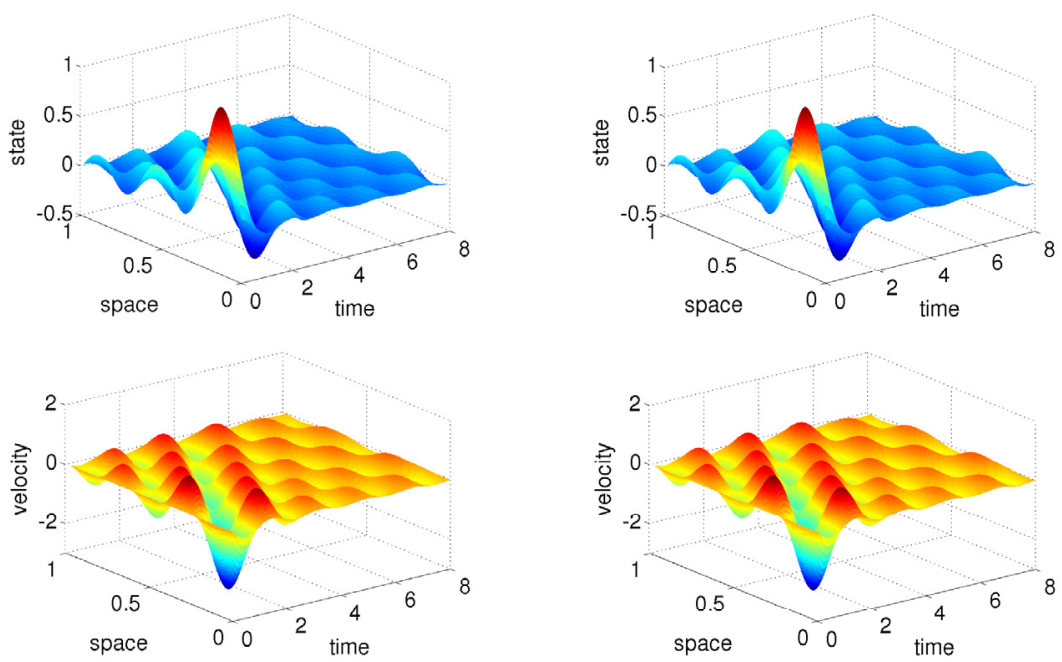

(a) HJB approach

(b) Riccati approach

Figure 2. State and velocity of the wave equation by the HJB and Riccati approach, $(\alpha=0.1)$. (In color online)

\subsubsection{Noise in the dynamics}

The noise enters the dynamics, i.e. we consider

$$
y_{t}=\mathbf{F}_{h}(y, u)+\mathbf{M}_{h}^{-1} \mathbf{B}_{h} \delta, \quad \delta=\left(\delta_{1}, \ldots, \delta_{5}\right)^{T}
$$

with random functions $\delta_{i}:[0, T] \rightarrow\left[\delta_{a}, \delta_{b}\right], i=1, \ldots, 5$, for $\delta_{a}, \delta_{b} \in \mathbb{R}$. The value function is, of course, computed for the dynamical system without noise and the feedback law is applied to the state resulting from the perturbed system. For data as in (7.1) we compute the numerical solution of (7.3) for noise scaled to the following interval

$$
\delta_{i}(s) \in[-4,4], \quad i=1, \ldots, 5, \quad s \in(0, T] .
$$



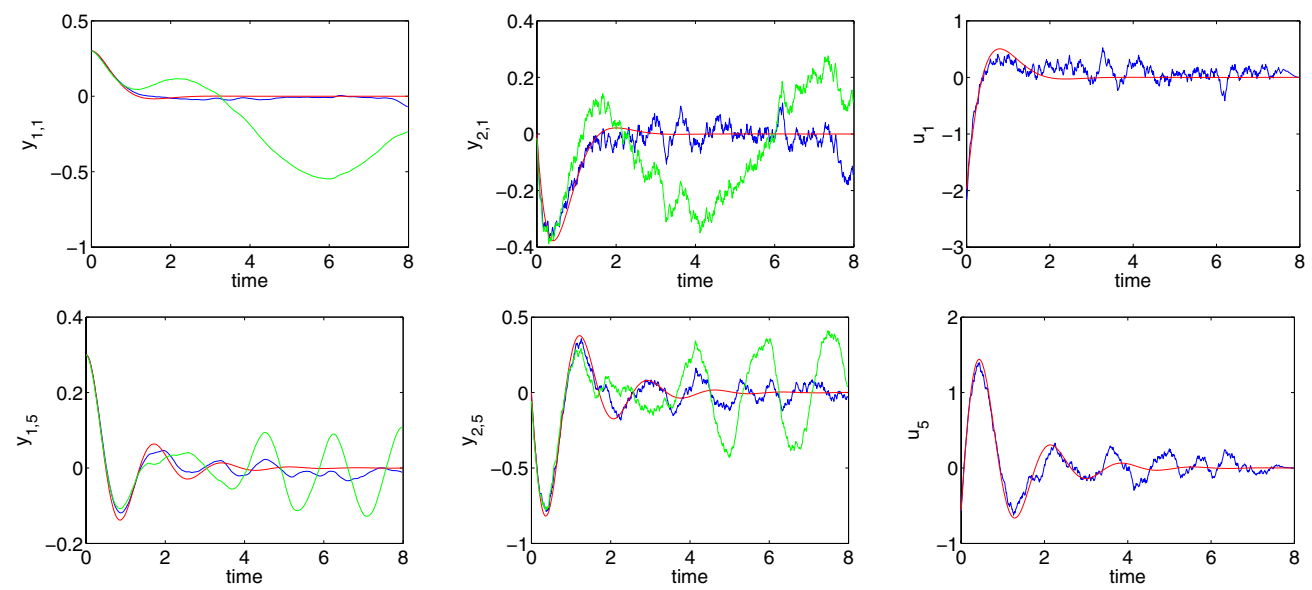

FiguRE 3. Noise in the dynamics: components of the trajectory and the feedback control via the HJB (blue) approach for the problem with noise and via the Riccati approach (red) for the equation without noise as well as the components of the trajectory (green) when applying the optimal control of the noise free system to the perturbed system, $(\alpha=0.01)$. (In color online)

For solving the HJB equation we proceed as in the previous example. The components of the corresponding trajectory and feedback control are presented in Figure 3. Again, the blue curves are the components of the trajectory computed via the HJB approach (the corresponding Riccati approach leads to quite similar results, why we omit the corresponding curves here). The red curves we obtain by the Riccati approach applied to the equation without noise. If we plug the resulting control in the perturbed system we obtain the green curves.

In contrast to the latter control we see that the feedback control stabilizes the dynamical system. The components $y_{2, i}, i=1, \ldots, 5$, are more susceptible to noise than $y_{1, i}$, since the noise enters the corresponding right hand sides.

In Figures $4 \mathrm{a}$ and $4 \mathrm{~b}$ the state and velocity for the HJB and Riccati approach are shown, respectively. Both approaches drive the state and velocity to zero. We compare these results with the state and velocity when applying the optimal control for the noise free dynamics to the perturbed system, see Figure 4c. In this case the control fails to drive state and velocity to zero.

\subsubsection{Noise in the observation}

The noise enters the observation, i.e. we consider the disturbed feedback law

$$
u(s)=\mathbf{K}^{\Delta}\left(x(s)+\delta(s) e_{10}, s\right), \quad e_{10}=(1, \ldots, 1)^{T}
$$

with noise $\delta(s):(0, T] \rightarrow \mathbb{R}$. In Figure 5 we see the components of the trajectory and the control. Although we apply the feedback operator on the perturbed state the resulting control stabilizes the trajectories. Numerical examples show that the amplitude of the oscillations increase with the amplitude of the noise. We have to choose the noise level smaller than in the first example namely $\delta(s) \in[0.01,0.01]$. The noise has a much stronger effect on the trajectory than in the previous examples. As in the previous example the HJB and Riccati approach for the system with noise lead to very similar curves.

\subsection{Linear wave equation - control of high order system}

In Example 3.1 we saw that we can expect a good approximation when discretizing the wave equation with 5 basis functions as we did in the previous examples. However, when considering nondiagonal operators 

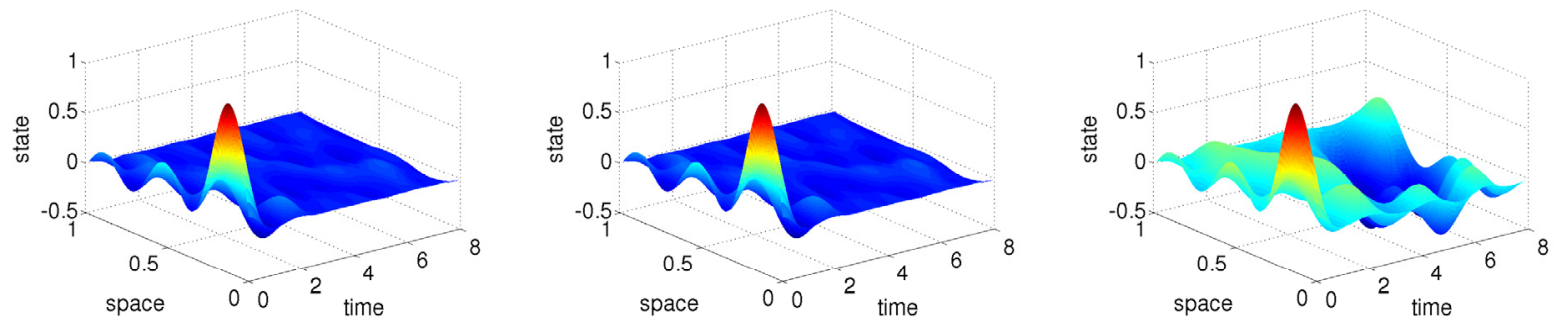

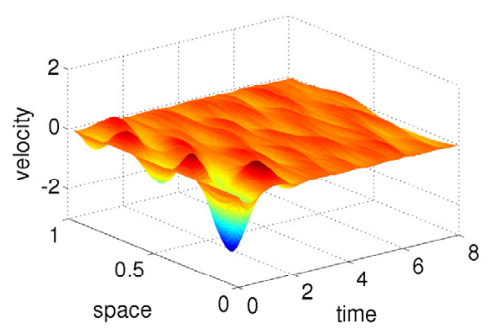

(a) HJB approach

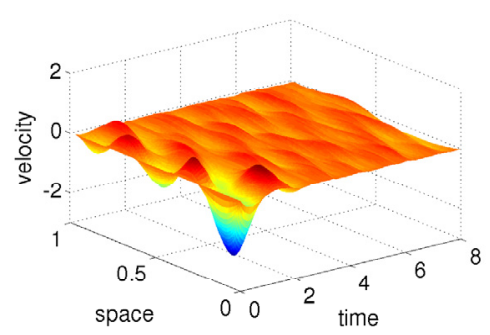

(b) Riccati approach

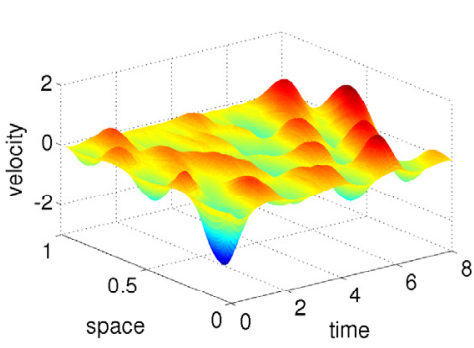

(c) Comparative solution

Figure 4. Noise in the dynamics: State and velocity of the wave equation via the HJB and Riccati approach as well as the state and velocity of a noisy dynamical system controlled by the optimal control of the noise free problem, $(\alpha=0.01)$. (In color online)
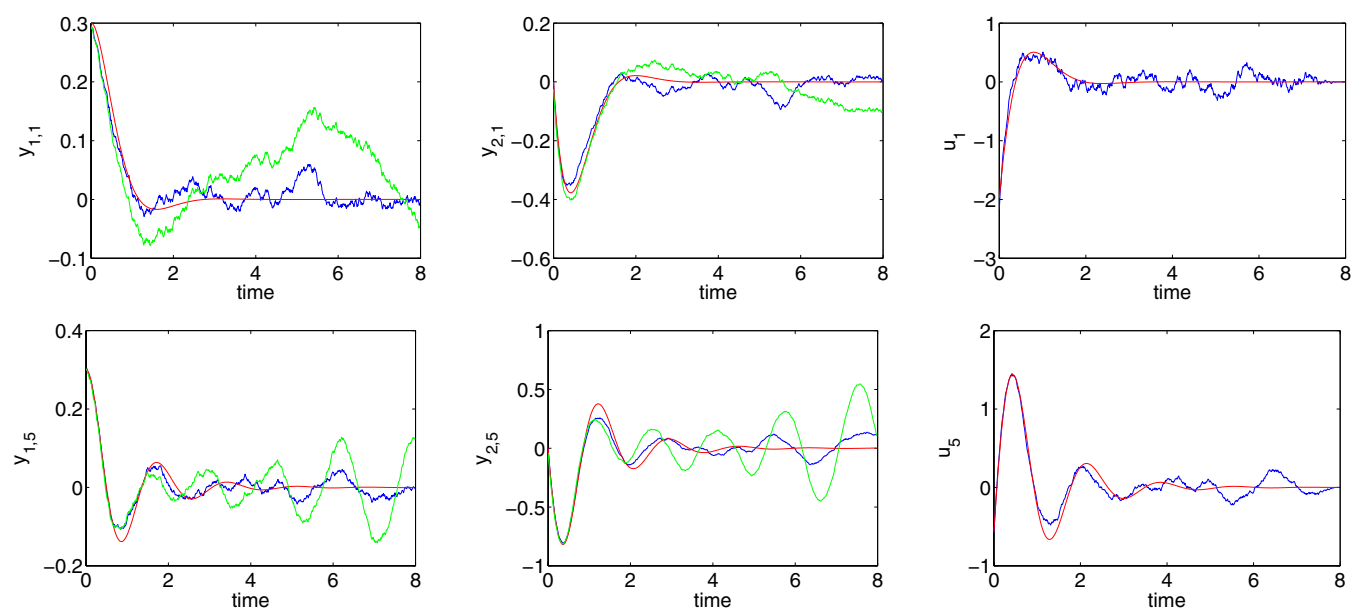

FIgURE 5. Noise in the observation: components of the trajectory and the feedback control via the HJB approach (blue) for the system with noise and via the Riccati approach (red) for the equation without noise as well as the components of the trajectory (green) when applying the optimal control of the noise free system to the perturbed system, $(\alpha=0.01)$. (In color online)

$\mathbf{B}_{h}$ or nonlinear problems the decomposition in subproblems is no longer possible since the equations of the corresponding dynamical system are coupled. In these cases we restrict the discretization to 3 basis functions for the state and for the velocity, because of the curse of dimensionality, which results in a six dimensional dynamical system. Nevertheless we can use the feedback control of this low order system to control a corresponding high order system. In the following we present an example, where we see the effects of the feedback control obtained from a low order system on a high order one. 
For the high order system we use a finite element discretization. For given $\tau>0$ we define the spatial mesh

$$
x_{i}=i \tau
$$

with $i=0, \ldots, q, q=1 / \tau$ and consider a standard linear finite element space

$$
V_{\tau}^{H}=\left\{\phi \in C_{0}(\bar{\Omega})|\phi|_{I_{q}} \in \mathcal{P}_{1}\left(I_{q}\right), I_{q}=[i \tau,(i+1) \tau], i=0, \ldots, q-1\right\},
$$

where $\mathcal{P}_{1}\left(I_{q}\right)$ consist of linear functions on $I_{q}$. Further we define the stiffness matrix $A^{\text {FEM }}$ and mass matrix $M^{\mathrm{FEM}}$ as in (3.2) with respect to the finite element basis and the corresponding discrete dynamics $\mathbf{F}^{\mathrm{FEM}}$ as in $(3.3)$ with $g_{h} \equiv 0$. Then the semi-discrete system is given by

$$
\left\{\begin{array}{l}
\frac{\mathrm{d}}{\mathrm{d} s} y_{x, t}^{\tau}=\mathbf{F}_{\tau}^{\mathrm{FEM}}\left(y_{x, t}^{\tau}, u^{\tau}\right)+\left(0, M^{\mathrm{FEM}}\right)^{T} \delta(s) e, \quad s \in(0, T], \\
y_{x, t}^{\tau}(0)=x
\end{array}\right.
$$

with noise $\delta:[0, T] \rightarrow \mathbb{R}, e \in \mathbb{R}^{q-1}, e(i)=1$ for $i=1, \ldots, q-1$, and initial point $x \in \mathbb{R}^{q-1}$. For the time discretization we apply a Crank-Nicolson scheme and use the same temporal mesh as for the low order system.

To set up an algorithm to compute the feedback control for the full order system we introduce the projection on the low dimensional space

$$
P^{L}: V_{\tau}^{H} \rightarrow \mathbb{R}^{3}, \quad \phi \mapsto P^{L} \phi
$$

with

$$
\left(P^{L} \phi\right)_{i}=2 \int_{\Omega} \phi(x) \sin (i \pi x) \mathrm{d} x, \quad i=1,2,3
$$

and the pointwise evaluation operator

$$
E: C(\bar{\Omega}) \rightarrow V_{\tau}^{H}, \quad E(\phi)=\bar{\phi}, \quad \bar{\phi}_{i}=\phi\left(x_{i}\right), \quad i=1, \ldots, q-1, \quad \phi \in C(\bar{\Omega}) .
$$

The feedback rule for the full order system is presented in Algorithm 1.

For the numerical example we choose the data as follows

$$
c=0.025, \quad T=8, \quad y_{0}(z)=(1.5 \sin (\pi z)+\sin (2 \pi z)+\sin (3 \pi z), 0)^{T}, \quad z \in \Omega
$$

with $\alpha=0.1$. We solve the HJB equation on $\mathcal{K}=[-2.5,2.5]^{6}$ with $\Delta t=2 \times 10^{-3}$. For the discretization in space we choose a spatial mesh with the following numbers of discretization points

$$
4 \times 8 \times 20 \times 4 \times 8 \times 20 .
$$

For the discretization of the wave equation we set the temporal step size to $k_{\mathrm{ds}}=0.02$ and $\tau=1 / 32$.

In Figure 6 we compare the components of the trajectory and control for the HJB and Riccati approach. For the chosen discretization of the HJB equation we observe very similar results for both approaches. In Figure 7 we see the optimal state and velocity of the low order system and the full order system without noise. As one can expect the results are the same. For the case with noise $\delta(s) \in[-10,10], s \in(0, T]$, we see in Figure 8 the state and velocity for the uncontrolled full order system and when applying the feedback control. In contrast to the uncontrolled case the state and velocity are stabilized by the feedback control.

If the initial data of the full order problem has components not lying in the low dimensional subspace they cannot be controlled as we see in Figure 9 where we choose the initial data as

$$
y_{0}(z)=(1.5 \sin (\pi z)+\sin (2 \pi z)+\sin (3 \pi x)+\sin (4 \pi z), 0)^{T}, \quad z \in \Omega .
$$

We observe that the component of the solution with respect to the fourth basis function remains in the system. 


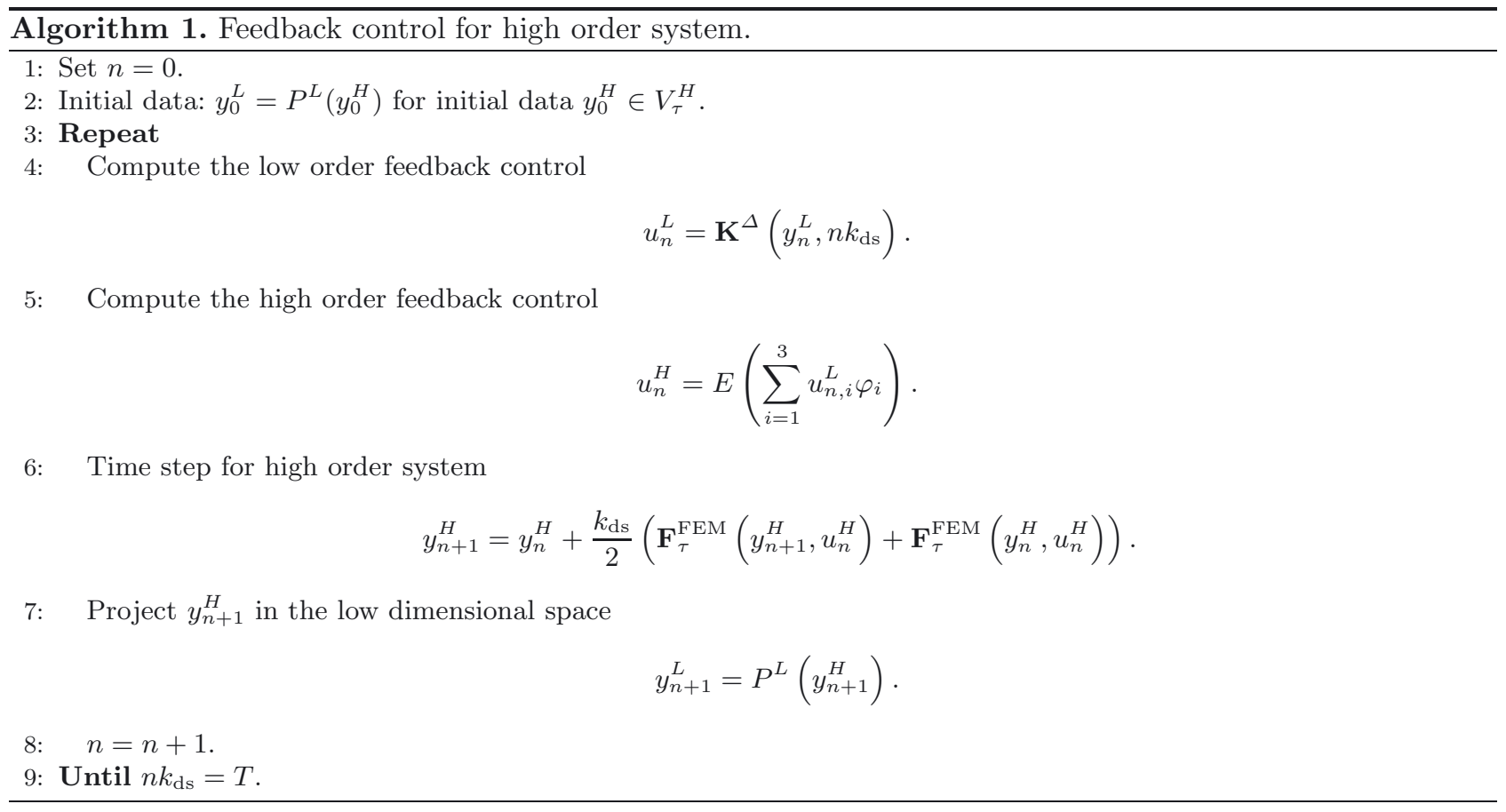

Next, let the control act only on an open subset $\omega$ of $\Omega$, i.e.

$$
\mathbf{B}^{\omega}=\chi_{\omega} \mathbf{B}
$$

with characteristic function $\chi_{\omega}$ of $\omega$. We choose $\omega=(0,0.75)$ and obtain a discrete operator given by

$$
\mathbf{B}_{h}^{\omega}=\left(0, B_{2}\right)^{T}, \quad B_{2}=\left(\begin{array}{ccc}
0.455 & 0.075 & -0.079 \\
0.075 & 0.375 & 0.135 \\
-0.079 & 0.135 & 0.349
\end{array}\right)
$$

This leads to a dynamics given by

$$
\mathbf{F}_{h}(x, u)=\mathbf{M}_{h}^{-1}\left(\mathbf{A}_{h} x+\mathbf{B}_{h}^{\omega} u\right) .
$$

The functions $p_{i}(x)=\chi_{\omega} \sin (i \pi x), i=1,2, \ldots$, are not eigenfunctions of the Laplacian. Therefore, since in every time step we project the state in the low dimensional space there will always remain components which cannot be controlled as wee see in Figure 10 where the state, velocity and control for the low and the full order system are shown for data as given (7.7).

\subsection{Semilinear wave equation - with noise}

In this example we consider an optimal control problem for a semilinear wave equation. Let the dynamics $\mathbf{F}$ be given as in (2.8) with

$$
g(y)=\frac{1}{2}\left(0, y^{3}\right)^{T}, \quad y \in L^{2}(\Omega) .
$$

Since we consider the HJB equation on a bounded domain we can without loss of generality replace $g$ by a Lipschitz continuous function satisfying (2.2). We set $c=0.025, T=4$, and $\alpha=0.1$. Using (3.1) for $N=3$ we have for the nonlinear term

$$
\int_{\Omega} g_{h, 2}\left(y_{1}^{h}(x, t)\right)^{T} \varphi(x) \mathrm{d} x=\mathbf{N}\left(y_{1}^{h}\right)(t), \quad \varphi=\left(\varphi_{1}, \varphi_{2}, \varphi_{3}\right)^{T},
$$



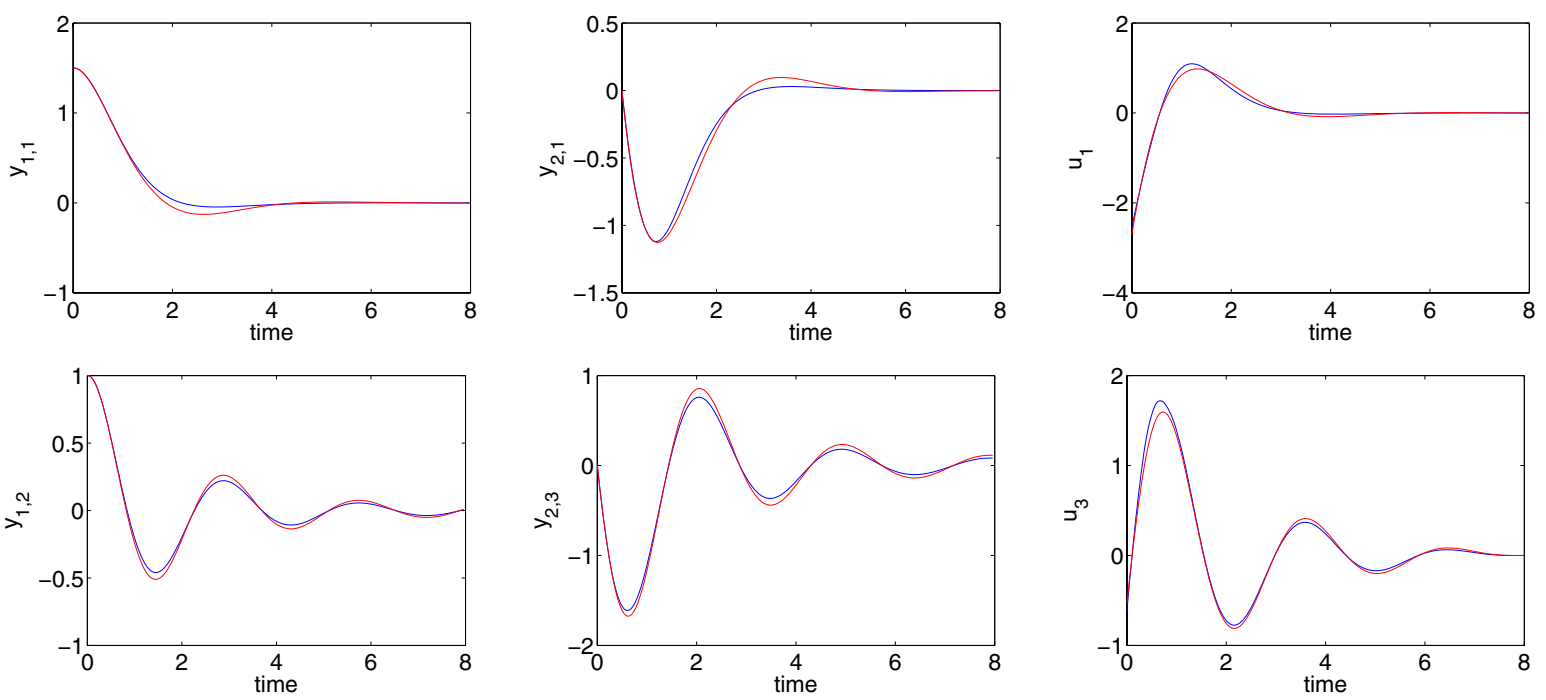

FiguRE 6. Components of the trajectory and the control of the HJB (blue curves) and Riccati (red curves) approach. (In color online)
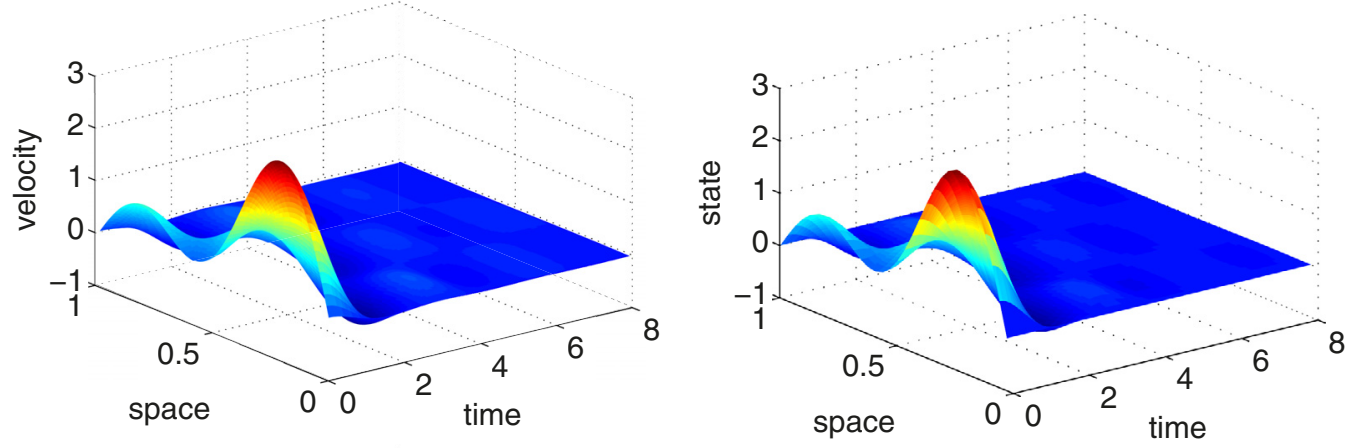

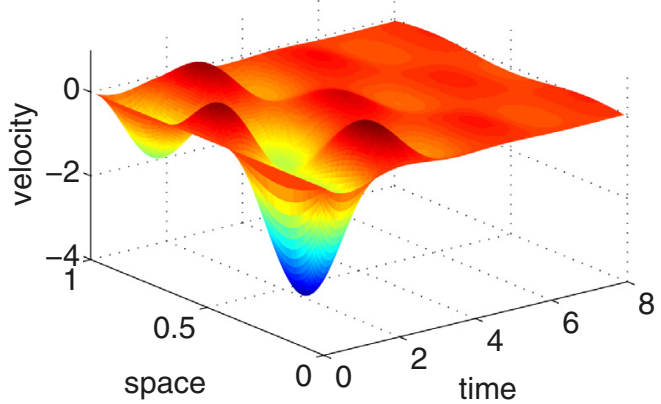

(a) Low order system

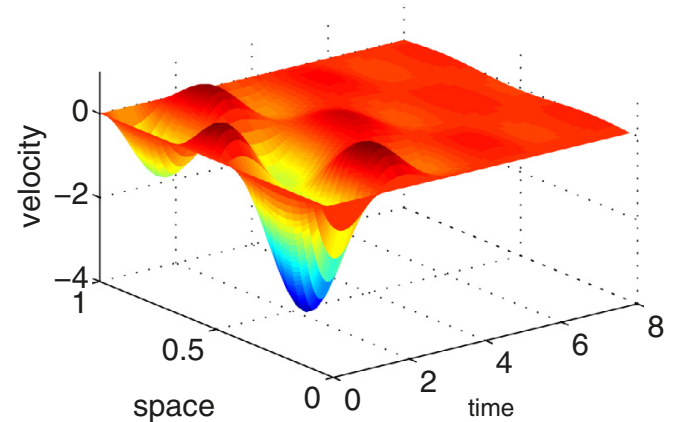

(b) High order system

FIGURE 7. State and velocity for low order system and high order system without noise in the dynamics. (In color online) 

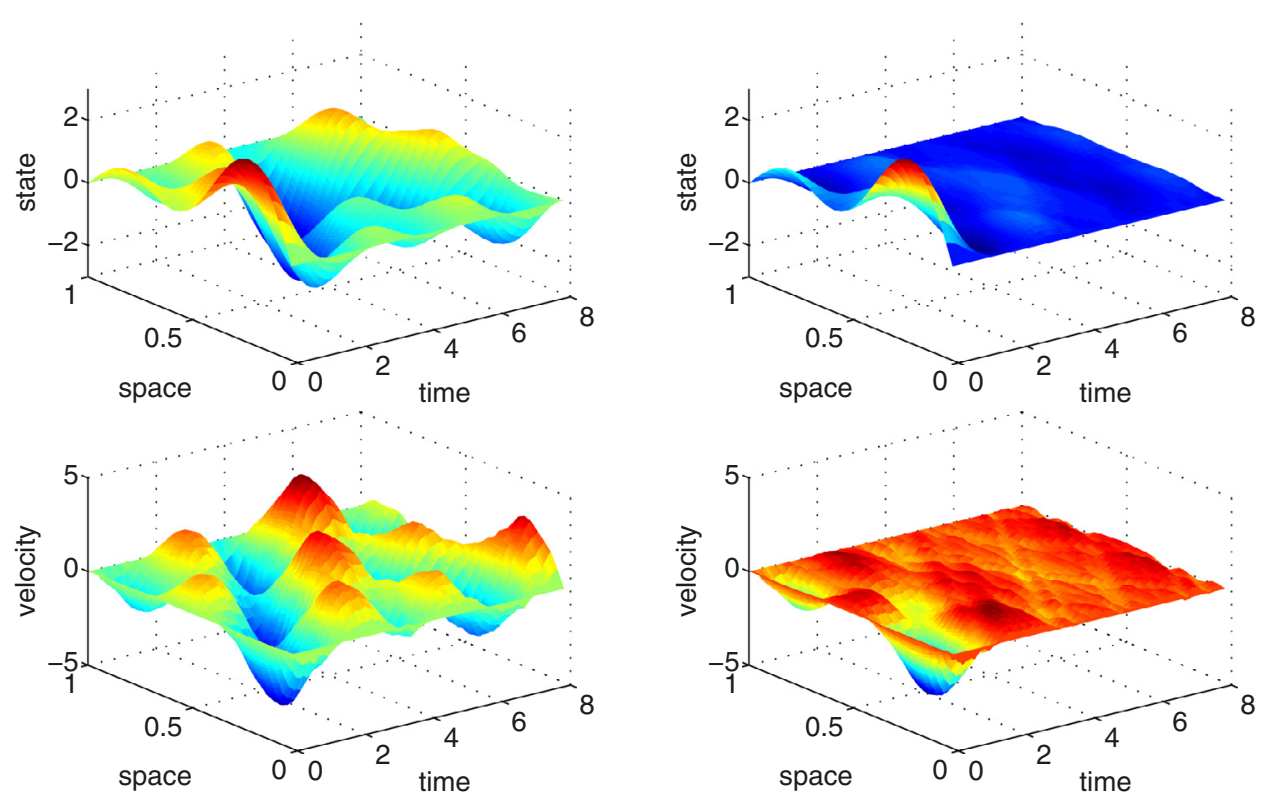

(a) Uncontrolled high order system with noise

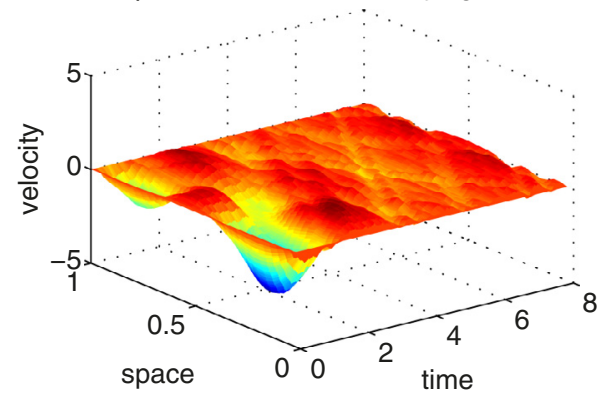

(b) Controlled high order system with noise

FiguRE 8. Uncontrolled and controlled state and velocity for high order system with noise in the dynamics. (In color online)

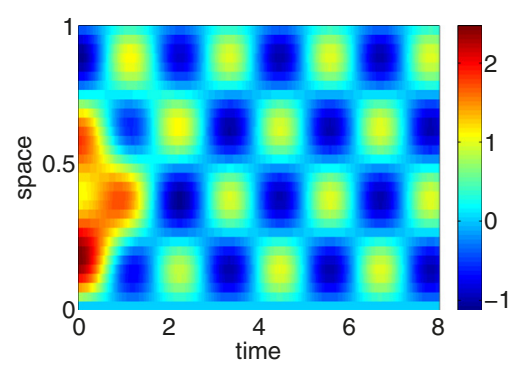

(a) State

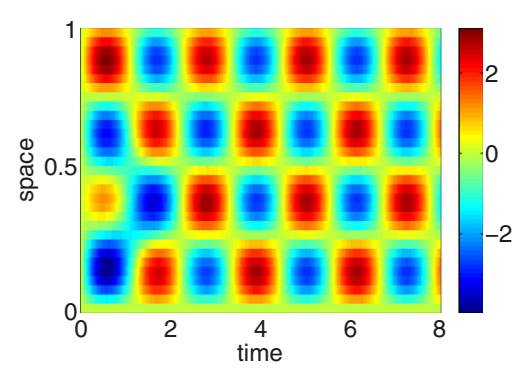

(b) Velocity

FIGURE 9. State and velocity of the high order system with initial data $x(z)=(1.5 \sin (\pi z)+$ $\sin (2 \pi z)+\sin (4 \pi z), 0)^{T}$ not lying in the low order subspace. (In color online)

that

$$
\mathbf{N}\left(y_{1}^{h}\right)=\frac{1}{2}\left(\begin{array}{c}
\frac{3}{8} y_{1,1}^{3}-\frac{3}{8} y_{1,1}^{2} y_{1,3}+\frac{3}{4} y_{1,1} y_{1,2}^{2}+\frac{3}{4} y_{1,1} y_{1,3}^{2}+\frac{3}{8} y_{1,2}^{2} y_{1,3} \\
\frac{3}{4} y_{1,1}^{2} y_{1,2}+\frac{3}{4} y_{1,1} y_{1,2} y_{1,3}+\frac{3}{8} y_{1,2}^{3}+\frac{3}{4} y_{1,2} y_{1,3}^{2} \\
-\frac{1}{8} y_{1,1}^{3}+\frac{3}{4} y_{1,1}^{2} y_{1,3}+\frac{3}{8} y_{1,1} y_{1,2}^{2}+\frac{3}{4} y_{1,2}^{2} y_{1,3}+\frac{3}{8} y_{1,3}^{3}
\end{array}\right)
$$

with $y_{1}^{h}=\left(y_{1,1}, y_{1,2}, y_{1,3}\right)^{T}$. We compare the results with a linearized Riccati approach. We solve the differential Riccati equation for the linearized state equation ( $c f$. Sect. 6), i.e. we consider

$$
z_{t}-\mathbf{M}_{h}^{-1} \mathbf{A}_{h} z-\mathbf{M}_{h}^{-1} 3 \bar{y}^{2} z=\mathbf{M}_{h}^{-1} \mathbf{B}_{h} u .
$$

and linearize around the target $\bar{y}=0$. 


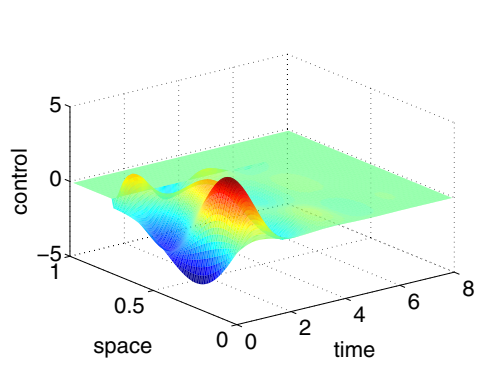

(a) Control
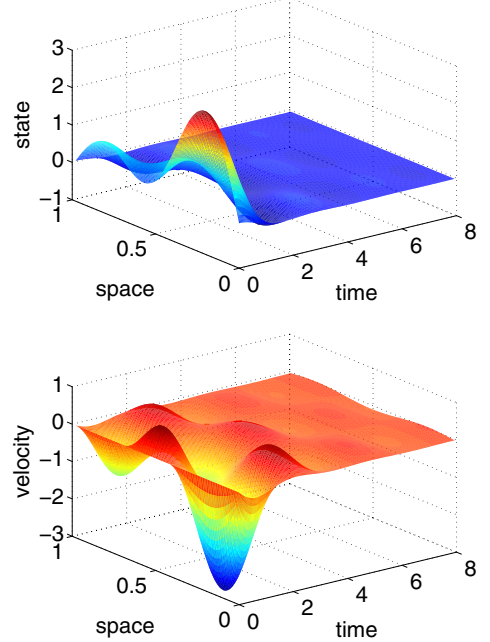

(b) Low order system - state, velocity
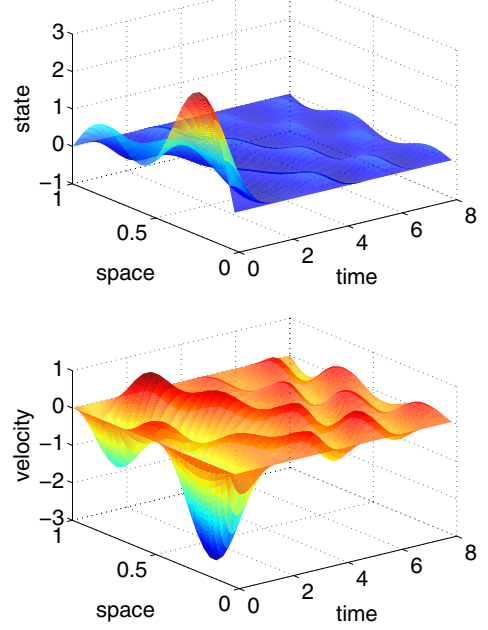

(c) High order system - state, velocity

FiguRE 10. Local control of the low and high order system. (In color online)

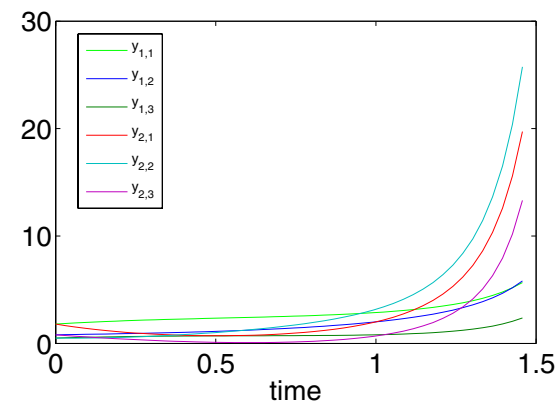

(a) Riccati approach

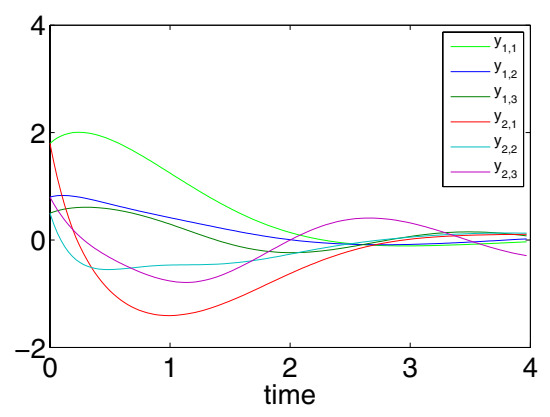

(b) HJB approach

FIGURE 11. Components of the trajectory for the linearized Riccati approach and HJB approach for nonlinear dynamics with initial point $y_{0}=\tilde{y}_{0}$. (In color online)

For the discretization of the HJB equation we choose the computational domain as

$$
[-2,2] \times[-1,1] \times[-1,1] \times[-4,4] \times[-1,1] \times[-1,1]
$$

and the numbers of discretization points as

$$
10 \times 8 \times 16 \times 10 \times 8 \times 16
$$

For time discretization we set $\Delta t=3.2 \times 10^{-3}$. For the discretization of the wave equation we choose $k_{\mathrm{ds}}=3.2 \times 10^{-2}$. 


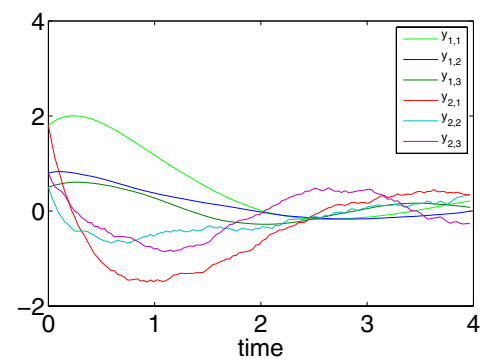

FiguRE 12. Components of the trajectory for the HJB approach for nonlinear dynamics with noise. (In color online)
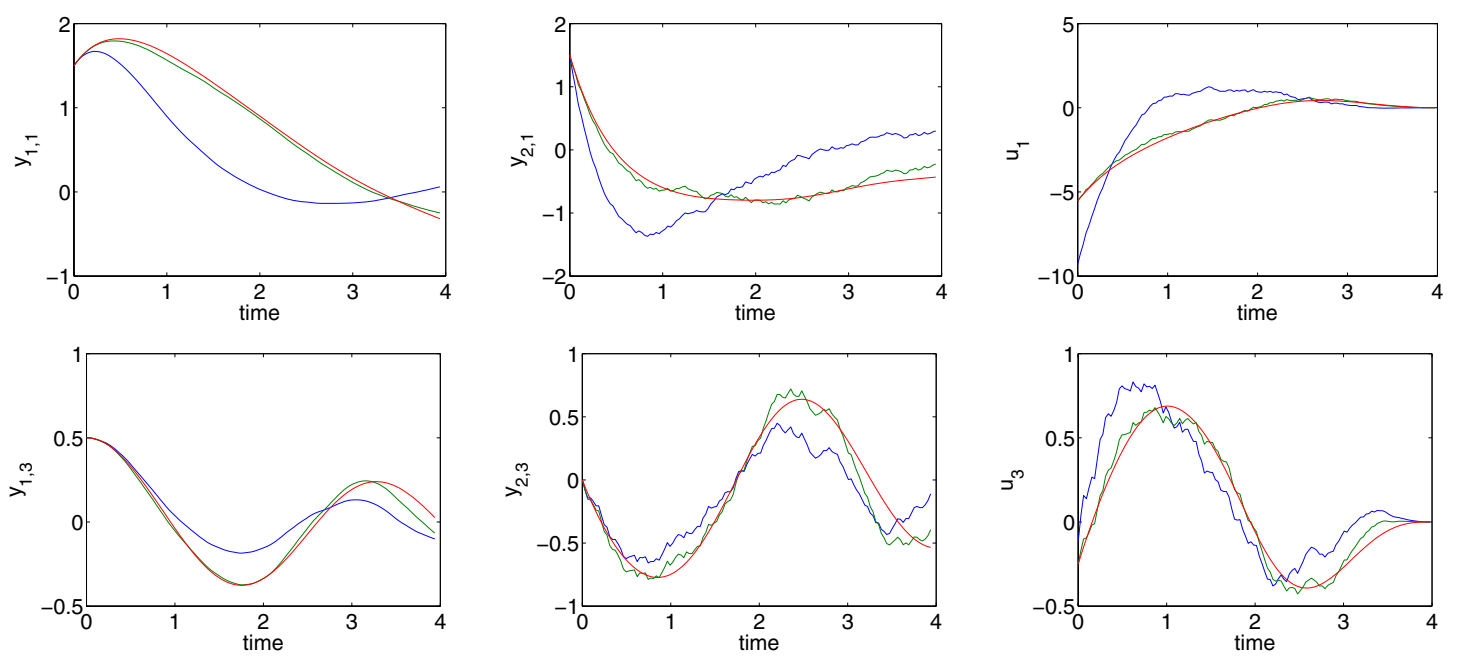

Figure 13. Components of the trajectories and the feedback control for the HJB (blue) and linearized Riccati approach with (green) and without (red) noise for nonlinear dynamics with initial point $y_{0}=\hat{y}_{0}$. (In color online)

We consider two different initial values

$$
\begin{aligned}
& \tilde{y}_{0}(z)=\left(\begin{array}{c}
1.8 \sin (\pi z)+0.8 \sin (2 \pi z)+0.5 \sin (3 \pi z) \\
1.8 \sin (\pi z)+0.5 \sin (2 \pi z)+0.8 \sin (3 \pi z)
\end{array}\right), \\
& \hat{y}_{0}(z)=\left(\begin{array}{c}
1.5 \sin (\pi z)+0.5 \sin (2 \pi z)+0.5 \sin (3 \pi z) \\
1.5 \sin (\pi z)
\end{array}\right)
\end{aligned}
$$

for $z \in \Omega$.

\subsubsection{Stabilization via HJB approach for initial data $\tilde{y}_{0}$ (linearized Riccati approach fails)}

The linearized Riccati approach fails to stabilize the system for initial data $\tilde{y}_{0}$. It leads to a blow up in finite time as we can see in Figure 11a. This is due to the fact that the initial data is not in the set $[-1,1]^{6}$, which leads to a stronger dominance of the nonlinear term. Nevertheless, the HJB approach stabilizes the system, see Figure 11b.

For noise $\delta_{i}:[0, T] \rightarrow[-3,3], i=1,2,3$, effecting the dynamics as

$$
y_{t}=\mathbf{F}_{h}(y, u)+\mathbf{M}_{h}^{-1} \mathbf{B}_{h} \delta, \quad \delta=\left(\delta_{1}, \delta_{2}, \delta_{3}\right)^{T}
$$

Figure 12 shows the components of the trajectory. 


\subsubsection{Stabilization via HJB approach for initial data $\hat{y}_{0}$ (linearized Riccati approach works)}

Finally, we consider the problem with noise in the dynamics for initial point $\hat{y}_{0}$. The components of the trajectory are shown in Figure 13. For $t>2.5$ the state is already close to zero and in particular smaller than 1 which implies that the linear term becomes dominant and the nonlinear very small. The influence of the nonlinear term is hence very small and thus the two feedback laws lead to similar curves. Thus in this case the initial point is sufficiently close to zero so that the Riccati approach still works.

\section{REFERENCES}

[1] A. Alla and M. Falcone, An adaptive POD approximation method for the control of advection-diffusion equations. Control and Optimization with PDE Constraints. Edited by C. Kunisch, K. von Winckel, G. Bredies, K. Clason. In vol. 164 of Int. Ser. Numer. Math. Springer (2013).

[2] A. Altarovici, O. Bokanowski and H. Zidani, A general Hamilton-Jacobi framework for non-linear state-constrained control problems. ESAIM: COCV 19 (2013) 337-357.

[3] H.T. Banks and K. Kunisch, The linear regulator problem for parabolic systems. SIAM J. Control. Optim. 22 (1984) $499-515$.

[4] E. Bänsch and P. Benner, Stabilization of incompressible flow problems by Riccati-based feedback. Constrained Optimization and Optimal Control for Partial Differential Equations. Edited by G. Leugering, S. Engell, A. Griewank, M. Hinze, R. Rannacher, V. Schulz, M. Ulbrich and S. Ulbrich. Birkhäuser (2012) 5-20.

[5] V. Barbu, S.S. Rodrigues and A. Shirikyan, Internal exponential stabilization to a nonstationary solution for 3d Navier-Stokes equation, SIAM J. Control Optim. 49 (2011) 1454-1478.

[6] M. Bardi and I. Capuzzo-Dolcetta, Optimal Control and Viscosity Solutions of Hamilton-Jacobi-Bellman Equations. Birkhäuser, Boston (2008).

[7] O. Bokanowski, Y. Cheng and C.-W. Shu, A discontinuous Galerkin scheme for front propagation with obstacles. Numer. Math. (2013) 1-31.

[8] O. Bokanowski, E. Cristiani and H. Zidani, An efficient data structure and accurate scheme to solve front propagation problems. J. Sci. Comput. 42 (2010) 251-273.

[9] O. Bokanowski, A. Desilles and H. Zidani, ROC-HJ-Solver. A C++library for solving HJ equations (2013).

[10] O. Bokanowski, N. Forcadel and H. Zidani, A general Hamilton-Jacobi framework for non-linear state constrained control problems. SIAM J. Control Optim. 48 (2010) 4292-4316.

[11] O. Bokanowski, J. Garcke, M. Griebel and I. Klompmaker, An adaptive sparse grid semi-Lagrangian scheme for first order Hamilton-Jacobi Bellman Equations. J. Sci. Comput. 55 (2012) 575-605.

[12] O. Bokanowski, N. Megdich and H. Zidani, Convergence of a non-monotone scheme for Hamilton-Jacobi-Bellman equations with discontinuous initial data. Numer. Math. 115 (2010) 1-44.

[13] J.-M. Buchot, L. Thevenet and J.P. Raymond, Nonlinear feedback stabilization of a two dimensional Burgers equation. ESAIM: COCV 16 (2010) 929-955.

[14] C. Canuto, M.Y. Hussaini., A. Quarteroni and T.A. Zang, Spectral Methods: Evolution to Complex Geometries and Applications to Fluid Dynamics. Scientific Computation. Springer-Verlag, Berlin, Heidelberg (2007).

[15] M. Falcone and R. Ferreti, Semi-Lagrangian Approximation Schemes for Linear and Hamilton-Jacobi Equations. Appl. Math. Society for Industrial and Applied Mathematics (2014).

[16] R. Ferretti, Internal approximation schemes for optimal control problems in Hilbert spaces J. Math. Systems 7 (1997) 1-25.

[17] L. Gaudio and A. Quarteroni, Spectral element discretization of optimal control problems. Spectral and High Order Methods for Partial Differential Equations. Edited by J.S. Hesthaven and E.M. Rønquist. In vol. 76 of Lect. Notes in Comput. Sci. Engrg. Springer Berlin Heidelberg (2011) 393-401.

[18] M. Gerdts, G. Greif and H.J. Pesch, Numerical optimal control of the wave equation: Optimal boundary control of a string to rest in finite time. Math. Comput. Simul. 79 (2008) 1020-1032.

[19] J.S. Gibson, An analysis of optimal modal regulation: convergence and stability. SIAM J. Control. Optim. 19 (1981) 686-707.

[20] J.S. Gibson, A numerical algorithm for optimal feedback gains in high dimensional linear quadratic regulator problems. SIAM J. Control Optim. 29 (1991) 499-515.

[21] S. Gombao and J.-P. Raymond, Hamilton-Jacobi equations for control problems of parabolic equations. ESAIM: COCV 12 (2006) 311-349.

[22] D. Gottlieb and S.A. Orszag, Numerical Analysis of Spectral Methods: Theory and Applications. Capital City Press, Montpelier, Vermont, USA (1991).

[23] M. Gugat and G. Leugering, $\mathrm{L}^{\infty}$-norm minimal control of the wave equation: on the weakness of the bang-bang principle. ESAIM: COCV 14 (2008) 254-283.

[24] E. Hernández, D. Kalise and E. Otárola, Numerical approximation of the LQR problem in a strongly damped wave equation. Comput. Optim. Appl. 47 (2010) 161-178.

[25] E. Hernández, D. Kalise and E. Otárola, A locking-free scheme for the LQR control of a Timoshenko beam. Comput. Appl. Math. 235 (2011) 1383-1393.

[26] H. Ishii, Uniqueness of unbounded viscosity solution of Hamilton-Jacobi equations. Indiana Univ. Math. J. 33 (1984) $721-748$. 
[27] K. Ito, On the regularity of solutions of an operator Riccati equation arising in linear quadratic optimal control problems for hereditary differential systems. J. Math. Anal. Appl. 140 (1989) 396-406.

[28] M. Jensen and I. Smears, On the convergence of finite element methods for Hamilton-Jacobi-Bellman Equations. SIAM J. Numer. Anal. 51 (2013) 137-162.

[29] A. Kröner and K. Kunisch, A minimum effort optimal control problem for the wave equation. Comput. Optim. Appl. 57 (2014) $241-270$.

[30] A. Kröner, K. Kunisch and B. Vexler, Semi-smooth Newton methods for optimal control of the wave equation with control constraints. SIAM J. Control Optim. 49 (2011) 830-858.

[31] K. Kunisch, S. Volkwein and L. Xie, HJB-POD based feedback design for the optimal control of evolution problems. SIAM J. Appl. Dyn. Systems 4 (2004) 701-722.

[32] K. Kunisch and D. Wachsmuth, On time optimal control of the wave equation, its regularization and optimality system. ESAIM: COCV 19 (2013) 317-336.

[33] K. Kunisch and L. Xie, POD-based feedback control of the Burgers equation by solving the evolutionary HJB equation. Comput. Math. Appl. 49 (2005) 1113-1126.

[34] I. Lasiecka and R. Triggiani, Differential and Algebraic Riccati Equations with Applications to Boundary/Point Control Problems: Continuous Theory and Approximation Theory. In vol. 164. Springer-Verlag (1991) 160.

[35] J.-L. Lions and E. Magenes, Non-Homogeneous Boundary Value Problems and Applications. In vol. I. Springer-Verlag, Berlin (1972).

[36] V. Mehrmann and H. Xu, Explicit solutions for a Riccati equation from transport theory. SIAM J. Matrix Anal. Appl. 30 (2008) 1339-1357.

[37] S. Osher and C.-W. Shu, Essentially nonoscillatory schemes for Hamilton-Jacobi equations. SIAM J. Numer. Anal. 28 (1991) 907-922.

[38] A.T Patera, A spectral element method for fluid dynamics: Laminar flow in a channel expansion. J. Comput. Phys. 54 (1984) 468-488.

[39] J.P. Raymond, Feedback boundary stabilization of the two-dimensional Navier-Stokes equations. SIAM J. Control Optim. 45 (2005) 790-828.

[40] J.A. Sethian, Fast marching methods. SIAM Reviews 41 (1999) 119-235.

[41] E. Zuazua, Exact controllability for semilinear wave equations in one space dimension. Ann. Inst. Henri Poincaré, Section C 10 (1993) 109-129. 